\title{
Soft gamma-ray constraints on a bright flare from the Galactic Center supermassive black hole
}

\author{
G. Trap ${ }^{1,2}$, A. Goldwurm ${ }^{1,2}$, R. Terrier ${ }^{2}$, K. Dodds-Eden ${ }^{3}$, \\ S. Gillessen ${ }^{3}$, R. Genzel ${ }^{3}$, E. Pantin ${ }^{2,4}$, P.O. Lagage ${ }^{2,4}$, \\ P. Ferrando ${ }^{1,2}$, G. Bélanger ${ }^{5}$, D. Porquet $^{6}$, N. Grosso ${ }^{6}$, \\ F. Yusef-Zadeh ${ }^{7}$, F. Melia ${ }^{8}$
}

\begin{abstract}
Sagittarius $A^{\star}\left(\operatorname{Sgr} A^{\star}\right)$ is the supermassive black hole residing at the center of the Milky Way. It has been the main target of an extensive multiwavelength campaign we carried out in April 2007. Herein, we report the detection of a bright flare from the vicinity of the horizon, observed simultaneously in X-rays (XMM-Newton/EPIC) and near infrared $(V L T / \mathrm{NACO})$ on April $4^{\text {th }}$ for 1-2 h. For the first time, such an event also benefitted from a soft $\gamma$-rays (INTEGRAL/ISGRI) and mid infrared (VLT/VISIR) coverage, which enabled us to derive upper limits at both ends of the flare spectral energy distribution (SED). We discuss the physical implications of the contemporaneous light curves as well as the SED, in terms of synchrotron, synchrotron self-Compton and external Compton emission processes.
\end{abstract}

Key words: Black hole physics, Radiation mechanisms: non-thermal, Galaxy: center, Gamma rays: observations, Infrared: general, X-rays: general PACS: 04.70.Bw, 95.85.Gn, 95.85.Hp, 95.85.Nv, 95.85.Pw, 97.60.Lf, 98.35.Jk, 98.35.Mp 


\section{Introduction}

From the discovery of a compact radio source, $\mathrm{Sgr} \mathrm{A}^{\star}$, at the Galactic Center (GC) in 1974 (Balick \& Brown, 1974) to the near infrared (NIR) tracking of stars in Keplerian motion around $\mathrm{Sgr} \mathrm{A}^{\star}$ three decades later (Schödel et al., 2002 Ghez et al., 2003), the evidence for a $\sim 4 \times 10^{6} M_{\odot}$ black hole with very slow proper motion at the dynamical center of our galaxy (Reid et al., 2008) gradually piled up (see Melia, 2007, for a general review and references therein).

Yet, the long quest for the high energy emission pertaining to the black hole has only been achieved recently. Sgr $\mathrm{A}^{\star}$ was resolved as a notably dim $\left(2.4 \times 10^{33} \mathrm{erg} \mathrm{s}^{-1}, 2-10 \mathrm{keV}\right)$ and slightly extended $\left(1.4^{\prime \prime}\right)$ point source with the Chandra satellite in 1999 (Baganoff et al., 2003a). One year later, the same instrument witnessed the source exhibiting an X-ray flare for $\sim 3 \mathrm{~h}$ (Baganoff et al., 2001). A 10 min long substructure within the light curve of the eruption and light time travel arguments imply that this event took place close to the event horizon ( $<15 R_{\mathrm{S}}$ ). Many other detections of X-ray flares followed, either with XMM-Newton or Chandra (see e.g. Goldwurm et al., 2003a; Baganoff et al., 2003b; Porquet et al., 2003; Bélanger et al., 2005), and established that the duty cycle of the black hole is nearly one X-ray flare per day. The origin of these events is still unclear, in spite of all the efforts aimed at their monitoring in different energy ranges. In 2003, NIR flares from Sgr A* were indeed discovered with the VLT (Genzel et al., 2003), and later confirmed by the Keck (Ghez et al., 2004) and the HST (Yusef-Zadeh et al., 2006a). They occur more frequently than the X-ray ones (around four per day) and have been observed in many NIR atmospheric pass bands (H, K, L, M). Each new

1 Service d'Astrophysique (SAp) / IRFU / DSM / CEA Saclay - Bât. 709, 91191 Gif-sur-Yvette Cedex, France

2 AstroParticule \& Cosmologie (APC) / Université Paris VII / CNRS / CEA / Observatoire de Paris - Bât. Condorcet, 10, rue Alice Domon et Léonie Duquet, 75205 Paris Cedex 13, France, trap@apc.univ-paris7.fr

3 Max Planck Institut für Extraterretrische Physik (MPE) - 85748 Garching, Germany

4 Astrophysique Interactions Multi-échelles (AIM) / Université Paris VII / CEA / CNRS - Bât. 709, 91191 Gif-sur-Yvette Cedex, France

5 European Space Agency (ESA) / ESAC - P.O. Box 78, Villanueva de la Canada, 28691 Madrid, Spain

6 Observatoire astronomique de Strasbourg / Université de Strasbourg / CNRS / INSU - 11, rue de l'Université, 67000 Strasbourg, France

7 Department of Physics and Astronomy / Northwestern University, Evanston, Illinois 60208, USA

8 Department of Physics and Steward Observatory / The University of Arizona, Tucson, Arizona 85721, USA 
infrared flare has generally induced either spatial (Clénet et al., 2005), spectral (Eisenhauer et al., 2005, Ghez et al., 2005; Gillessen et al., 2006; Krabbe et al., 2006; Hornstein et al., 2007), polarimetric (Eckart et al., 2006b; Meyer et al., 2006, 2007; Trippe et al., 2007), or timing studies (Meyer et al., 2008; Do et al., 2009). Numerous multiwavelength campaigns showed that an X-ray flare always comes along with a simultaneous NIR one ${ }^{9}$ (Eckart et al., 2004 , 2006a, 2008a; Yusef-Zadeh et al., 2006a; Hornstein et al., 2007), and maybe a delayed submm one (Marrone et al., 2008; Eckart et al., 2008b; Yusef-Zadeh et al., 2008) caused by plasmon expansion (Liu et al., 2004; Yusef-Zadeh et al., 2006b).

Above $6 \mu \mathrm{m}$, in the mid infrared (MIR), no detection of Sgr $\mathrm{A}^{\star}$ has been reported so far. Recent upper limits on the black hole flux at $8.6 \mu \mathrm{m}$ were set by the VLT/VISIR instrument during low level NIR variability by Schödel et al. (2007), who argued that a detection would be reachable in case of a strong NIR flare.

Above $20 \mathrm{keV}$, repeated surveys of the heart of the Milky Way in soft $\gamma$-rays with the INTEGRAL satellite unveiled a persistent pointlike source compatible with Sgr A* location (within the 1' error radius), IGR J174562901 (Bélanger et al. 2004, 2006). The nature of the source is still uncertain, and a possible association with the supermassive black hole remains conceivable. Given the limited angular resolution of the soft $\gamma$-ray telescope INTE$G R A L /$ IBIS/ISGRI $\left(\sim 12^{\prime}\right.$ FWHM), the best way to unequivocally identify the mysterious IGR J17456-2901 with Sgr A* is the detection of correlated variability between soft $\gamma$-rays and other wavelengths.

To tackle the above puzzles and investigate the correlated X-ray/NIR variability of Sgr $\mathrm{A}^{\star}$ in more details, a coordinated multiwavelength campaign on the GC was conducted in spring 2007. It involved in particular the XMMNewton and INTEGRAL satellites for the high energies, as well as the VLT/ NACO and $V L T / V I S I R$ ground instruments to cover the NIR and MIR part of the spectrum, respectively. Their results are presented in Sect. 2 and interpreted in Sect. 3. Note that the X-ray and infrared findings have already been published by Porquet et al. (2008) and Dodds-Eden et al. (2009), respectively.

We will not discuss here the short term variability of Sgr A* in April 2007 at $\mathrm{cm}, \mathrm{mm}$, and submm wavelengths, which will be reported in another article, along with NIR results obtained by the Hubble Space Telescope (Yusef-Zadeh et al. 2009 10 .

$\overline{9}$ The converse is not true, some NIR flares have no X-ray counterpart Hornstein et al., 2007).

${ }^{10}$ For further discussion of the past variability of $\mathrm{Sgr} \mathrm{A}^{\star}$ in $\mathrm{cm}$ and $\mathrm{mm}$ bands, see for example Zhao et al. (2001); Herrnstein et al. (2004) and Tsuboi et al. (1999); Zhao et al. (2003), respectively. 
Throughout this paper we adopt a GC distance of $8 \mathrm{kpc}(\mathrm{Reid}, 1993)$ and a black hole mass $M_{\bullet}=4 \times 10^{6} M_{\odot}$ (Ghez et al. 2008), for which the Schwarzschild radius is $R_{\mathrm{S}}=1.2 \times 10^{12} \mathrm{~cm}$.

\section{Observations \& results}

\subsection{X-rays}

The XMM-Newton satellite (Jansen et al., 2001) was pointed towards the GC during $\sim 2.5$ consecutive revolutions, from March $30^{\text {th }}$ to April $4^{\text {th }} 2007$. The data of the EPIC/PN (Strüder et al., 2001) and EPIC/MOS1-2 cameras (Turner et al., 2001) were processed and analyzed through the procedure described in Porquet et al. (2008).

We produced an image of the last revolution of the campaign (rev-1340, $97.6 \mathrm{ks}$ exposure), cleaned for out of time events in the $2-10 \mathrm{keV}$ band (see Fig. 1, middle). Two GC transient X-ray binaries and bursters, active at the time, stand out prominently: GRS 1741.9-2853 (Trap et al., 2009) and AX J1745.6-2901 (Grosso et al., 2008). Sgr A* is clearly apparent in the middle since this observation contains several flares from the vicinity of the black hole, enhancing its average luminosity.

Indeed, on April $4^{\text {th }}$, a high level of flaring activity from Sgr $\mathrm{A}^{\star}$ was caught. A bright flare (Fig. 2) - the second brightest ever recorded $(\sim 100$ times the quiescent level) in the X-ray band (2-10 keV) — was rapidly followed by three moderate ones. The bright event lasted $\sim 1 \mathrm{~h}$; its $\mathrm{PN}$ light curve has a rather symmetrical morphology and no apparent substructures (Fig. 4). Note that the $10^{\prime \prime}$ radius area used to extract this light curve not only contains Sgr A* but other X-ray sources as well: a pulsar wind nebula candidate, G 359.95-0.04

(Wang et al., 2006), the star cluster IRS 13, and diffuse emission (Baganoff et al., 2003a), which all, however, provide a constant contribution.

From a spectral point of view, this outburst was rather soft. The best fit to the data with an absorbed power-law model, including dust scattering, yields the following parameters: a spectral photon index $\Gamma=2.3 \pm 0.3\left(N(E) \propto E^{-\Gamma}\right)$ and a column density $N_{\mathrm{H}}=12 \pm 2 \times 10^{22} \mathrm{~cm}^{-2}$. In Sect. 3, we will use an equivalent definition of the spectral index, $\beta_{\mathrm{X}}$, easier to compare to other multiwavelength spectra: $\beta_{\mathrm{X}}=-\Gamma+2=-0.3 \pm 0.3$ with $\nu F_{\nu}^{\mathrm{X}} \propto \nu^{\beta \mathrm{X}}$. The unabsorbed mean flux of the flare was $16 \pm 3 \times 10^{-12} \mathrm{erg} \mathrm{s}^{-1} \mathrm{~cm}^{-2}(2-10 \mathrm{keV})$, which translates to a luminosity of $2.4 \pm 4 \times 10^{35} \mathrm{erg} \mathrm{s}^{-1}$ at the GC distance. Albeit luminous relative to previously observed X-ray flares, it was still $\sim 9$ orders of magnitude below the Eddington luminosity for a supermassive black 


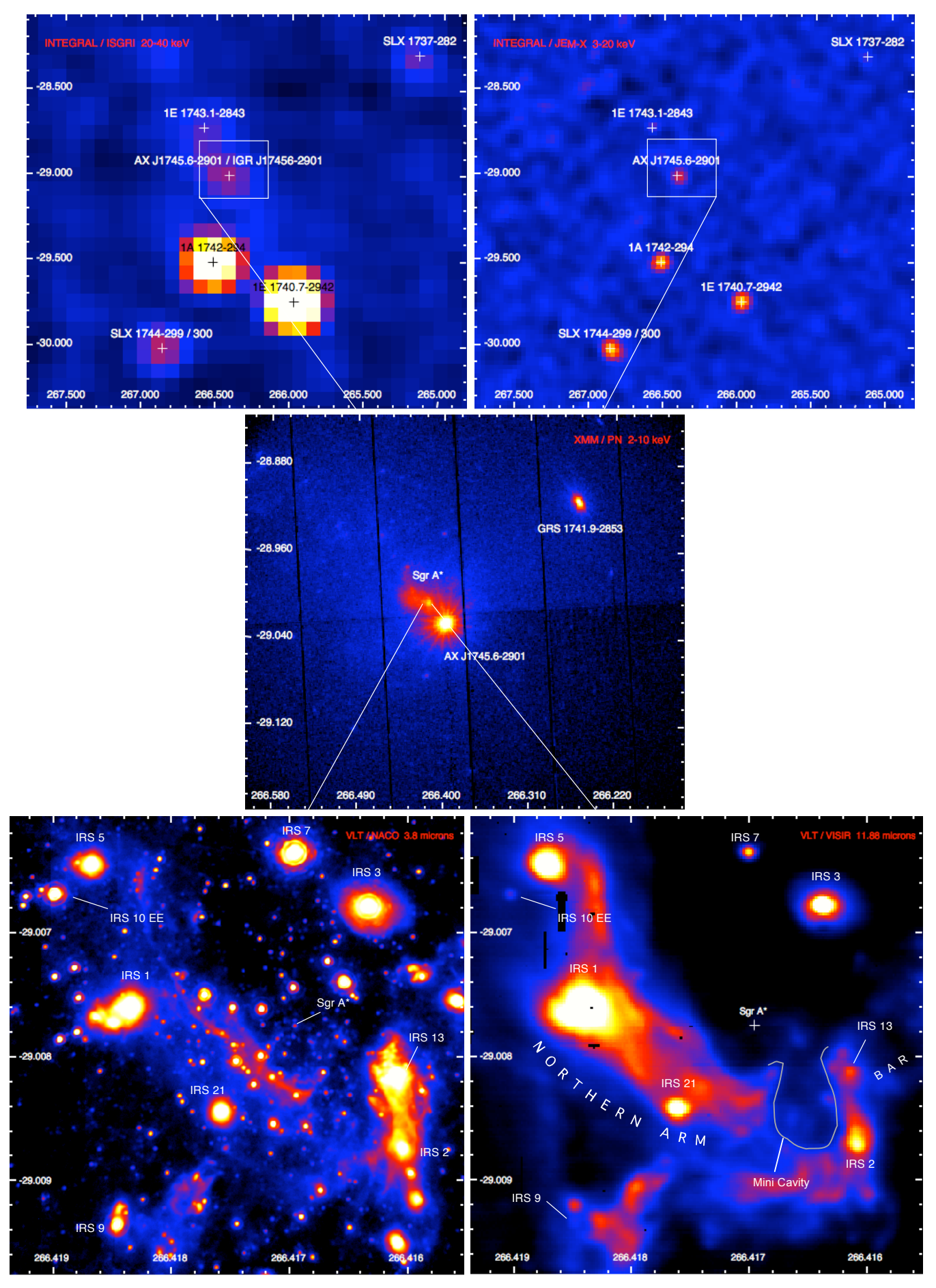

Fig. 1. Multiwavelength and multiscale views of the Galactic Center in April 2007 in R.A. $\left(^{\circ}\right)$ horizontally and Dec. $\left(^{\circ}\right)$ vertically (North and East point towards the top and the left, respectively). Top, left: INTEGRAL/ISGRI mosaic in the 20-40 keV band. The Galactic plane runs from upper left to bottom right. Top, right: INTEGRAL/JEM-X 1 mosaic in the 3-20 keV band. Middle: XMM/PN image in the $2-10 \mathrm{keV}$ band. Bottom, left: VLT/NACO image at the peak of the flaring period at $3.8 \mu \mathrm{m}$. Bottom, right: VLT/VISIR average image of the $3^{\text {rd }} / 4^{\text {th }}$ April night at $11.88 \mu \mathrm{m}$. The three dusty arms swirling around Sgr $\mathrm{A}^{\star}$ compose the so-called "mini-spiral". 


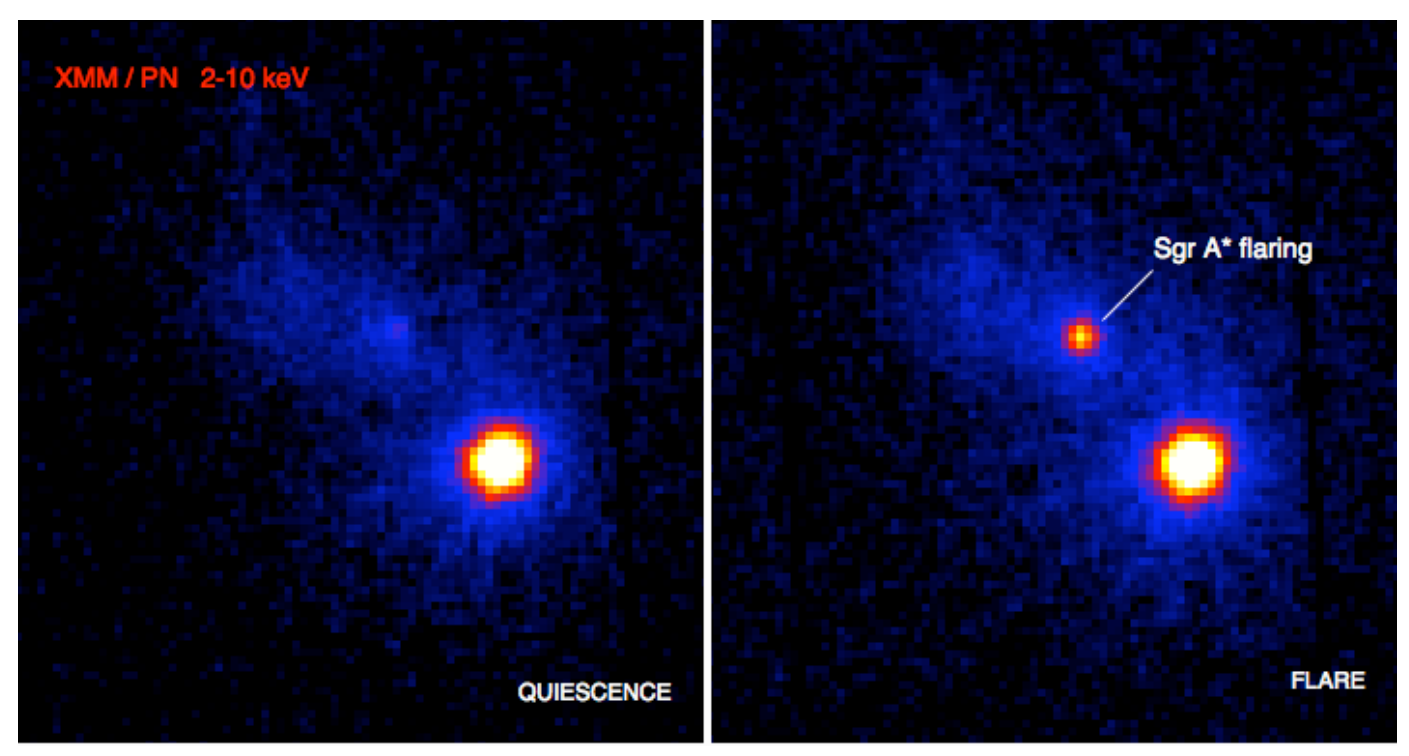

Fig. 2. Left: X-ray image of the quiescent phase during $50 \mathrm{~min}$ before the flare. Right: 50 min image during the flare.

hole of this kind.

As pointed out by Porquet et al. (2008), this rapid train of flares in just a few hours challenges disruption mechanisms of the accretion flow as the origin of the outbursts, since they rely on temporary storage of mass/energy. This energy should indeed be released at once during the outburst, with a radiation efficiency of a few percent. But, the weak accretion rate of the black hole seems insufficient $\left(\sim 10^{16-17} \mathrm{~g} \mathrm{~s}^{-1}\right.$, Melia (2007)) to accumulate the required energy on such short timescales. In contrast, scenarios based on the stochastic infall and tidal disruption of gas clumps (Tagger \& Melia, 2006; Falanga et al., 2007, 2008) or small bodies (Cadež et al., 2008) do not encounter this issue.

\subsection{Near infrared}

The $V L T / \mathrm{NACO}$ (NAOS+CONICA) set of instruments (Lenzen et al. 2003, Rousset et al., 2003) installed on the ESO/VLT unit telescope Yepun (UT4) at Paranal, Chile, observed the Galactic nucleus every nights from April $1^{\text {st }}$ to April $6^{\text {th }}$ in multiple NIR bands: L' $(3.8 \mu \mathrm{m}), \mathrm{K}_{\mathrm{S}}(2.1 \mu \mathrm{m})$, and $\mathrm{H}(1.6 \mu \mathrm{m})$. The details of the data reduction and analysis are presented in Dodds-Eden et al. (2009).

In particular, from 5:00 to 7:00 (UT) on April $4^{\text {th }}$, NACO followed the strong X-ray flare mentionned in Sect. 2.1 in the L' band. We constructed an image of the GC during this flare period (Fig. 1, bottom, left, and Fig. 3), in which Sgr $\mathrm{A}^{\star}$ is confused with the star S 17 and a small cloud of dust (Clénet et al., 2005). On Fig. 4, we display the light curve of the flare. Substructures 


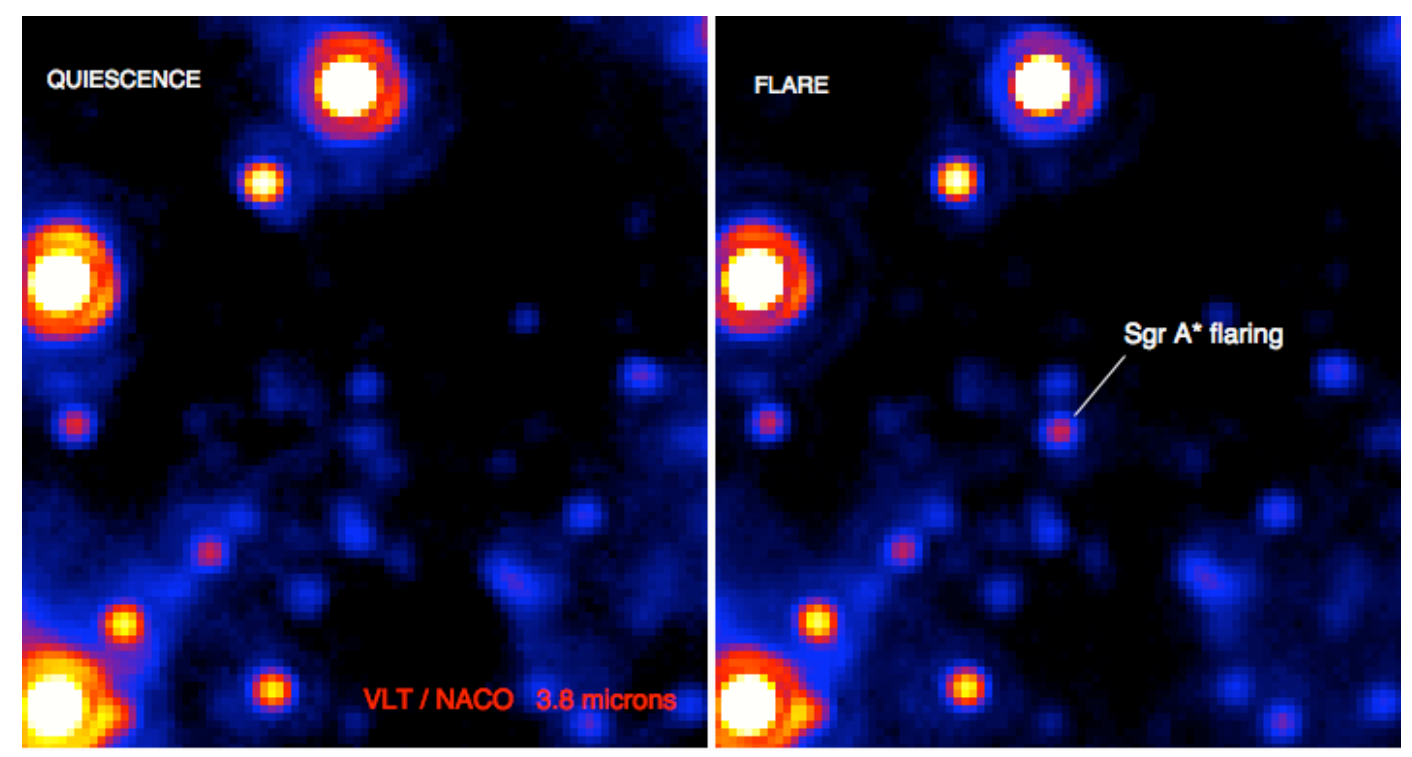

Fig. 3. Left: NIR image of the quiescent phase during six minutes before the flare. Right: Six minutes image at the apogee of the flare.

on a timescale of $\sim 20$ min within the light curve are evident. The shortest variation in the light curve $(\Delta t \sim 1 \mathrm{~min})$ constrains the emitting zone to a size no bigger than $c / \Delta t \sim 1.5 R_{\mathrm{S}}$. This is the first time that such features are visible in the L' band. Regarding the total duration of the eruption, it lasted distinctively longer in the NIR than in X-rays $(\sim 2 \mathrm{~h}$ vs. $\sim 1 \mathrm{~h})$, even though the X-ray background could hide the rising and decaying flanks of the flare. Note that the X-ray/NIR flare detected by Chandra/Keck on July $17^{\text {th }}$ 2006 (Hornstein et al., 2007) also indicated a longer NIR duration, even if Chandra has a smaller X-ray background than XMM-Newton thanks to its better point spread function (PSF). As for the peak of the flare, there is no time lag bigger than $\sim 3$ min between NIR wavelengths and X-rays.

Assuming an extinction $A_{\mathrm{L}}=1.8 \mathrm{mag}$, the NIR flare peaked at $\sim 30 \mathrm{mJy}$ (dereddened), which makes it one of the most powerful NIR flare ever captured, and definitely the brightest one detected simultaneously in X-rays. To allow comparison with other wavelengths, we also computed the background subtracted, extinction corrected, mean flux of the flare over the period of MIR observations (see Sect. 2.3): $19.1 \pm 3.6 \mathrm{mJy}$ at $3.8 \mu \mathrm{m}$. No direct NIR spectral information are available for this flare.

\subsection{Mid infrared}

VISIR, the VLT Imager and Spectrometer for the mid Infrared mounted on the ESO/VLT unit telescope Melipal (UT3) (Lagage et al., 2004; Pantin et al. 2005), pointed the GC from 2007-04-04 5:29:00 to 2007-04-04 10:34:00 
(UT) as part of a guaranteed time program. Data were acquired with the imager and PAH2_2 filter on, at $11.88 \pm 0.37 \mu \mathrm{m}$ in the atmospheric window N. The Small Field mode (SF) was employed, which resulted in a field of view of $256 \times 256$ pixels $\left(19.2^{\prime \prime} \times 19.2^{\prime \prime}\right)$, each pixel corresponding to $0.075^{\prime \prime} \times 0.075^{\prime \prime}$. Data reduction and analysis techniques are given in Dodds-Eden et al. (2009).

No point source at the position of $\operatorname{Sgr} \mathrm{A}^{\star}$ is detected in either the individual images or the collapsed image of the entire night (Fig. 1. bottom, right). We also performed a Lucy-Richardson deconvolution with HD 102461 as point spread function without success. The flux from a box of $0.375^{\prime \prime} \times 0.375^{\prime \prime}$ centered on the position of $\mathrm{Sgr} \mathrm{A}^{\star}$ is fairly constant (see the light curve on Fig. 4) with an average value of $123 \pm 6 \mathrm{mJy}$ (not dereddened). This flux may be attributed to the faint and diffuse dust ridge on which Sgr A* lies. Our measured value is consistent with previous VISIR observations (Eckart et al., 2004, Schödel et al., 2007) and other instruments before (Stolovy et al., 1996: Cotera et al., 1999: Morris et al., 2001).

However, for the first time, the measurements presented here were concurrent with a bright X-ray/NIR flare from Sgr $\mathrm{A}^{\star}$ as shown in Sect. 2.1 and 2.2. We estimate that Sgr $\mathrm{A}^{\star}$ could not have been brighter than $\sim 12$ mJy at $11.88 \mu \mathrm{m}(3 \sigma$, not dereddened). This value is compatible with VISIR empirical sensitivity at this wavelength: $7 \mathrm{mJy} / 10 \sigma / 1 \mathrm{~h}$ (median value for different atmospheric conditions). We note also that similar constraints were obtained with VISIR during NIR variability by Gillessen et al. (2006), Schödel et al. (2007), and Haubois et al. (2008).

The value of the extinction correction, $A_{\lambda}$, in the MIR depends critically on the strength and shape of the silicate absorption feature at $\sim 10 \mu \mathrm{m}$. In the literature, $A_{\lambda}$ is usually given as ratios relative to $A_{\mathrm{V}}$ or $A_{\mathrm{K}}$, so we use $A_{\mathrm{K}}=2.8 \mathrm{mag}\left(A_{\mathrm{V}}=25 \mathrm{mag}\right)$ to ensure consistency across our multiwavelength observations. The closest extinction measurement to $\lambda=11.88 \mu \mathrm{m}$ was made by Lutz (1999) for a wavelength of $\sim 12.4 \mu \mathrm{m}$. We consider the recent theoretical model of Chiar \& Tielens et al. (2006) for the extinction profile in the silicate region to allow us to extrapolate the value measured at $12.4 \mu \mathrm{m}$ to $11.88 \mu \mathrm{m}$. When normalized to the Lutz (1999) values, the model predicts $A_{11.88 \mu \mathrm{m}}=1.7 \pm 0.2 \mathrm{mag}$ and hence the dereddened $3 \sigma$ upper limit on the MIR emission of Sgr A* during the flare is $\sim 57 \mathrm{mJy}$. Schödel et al. (2007) found an upper limit of $\sim 22 \mathrm{mJy}$ at $8.6 \mu \mathrm{m}$ during quiescence of Sgr $\mathrm{A}^{\star}$ and predicted a bright flare was likely to come out of the noise in the MIR. Yet, using the dereddening used here, their limit would be a bit higher, $~ 32 \mathrm{mJy}$, and, since we did not detect this strong X-ray/NIR flare, we speculate that no flaring counterpart of Sgr $A^{\star}$ will be detected with the current settings of VISIR. 


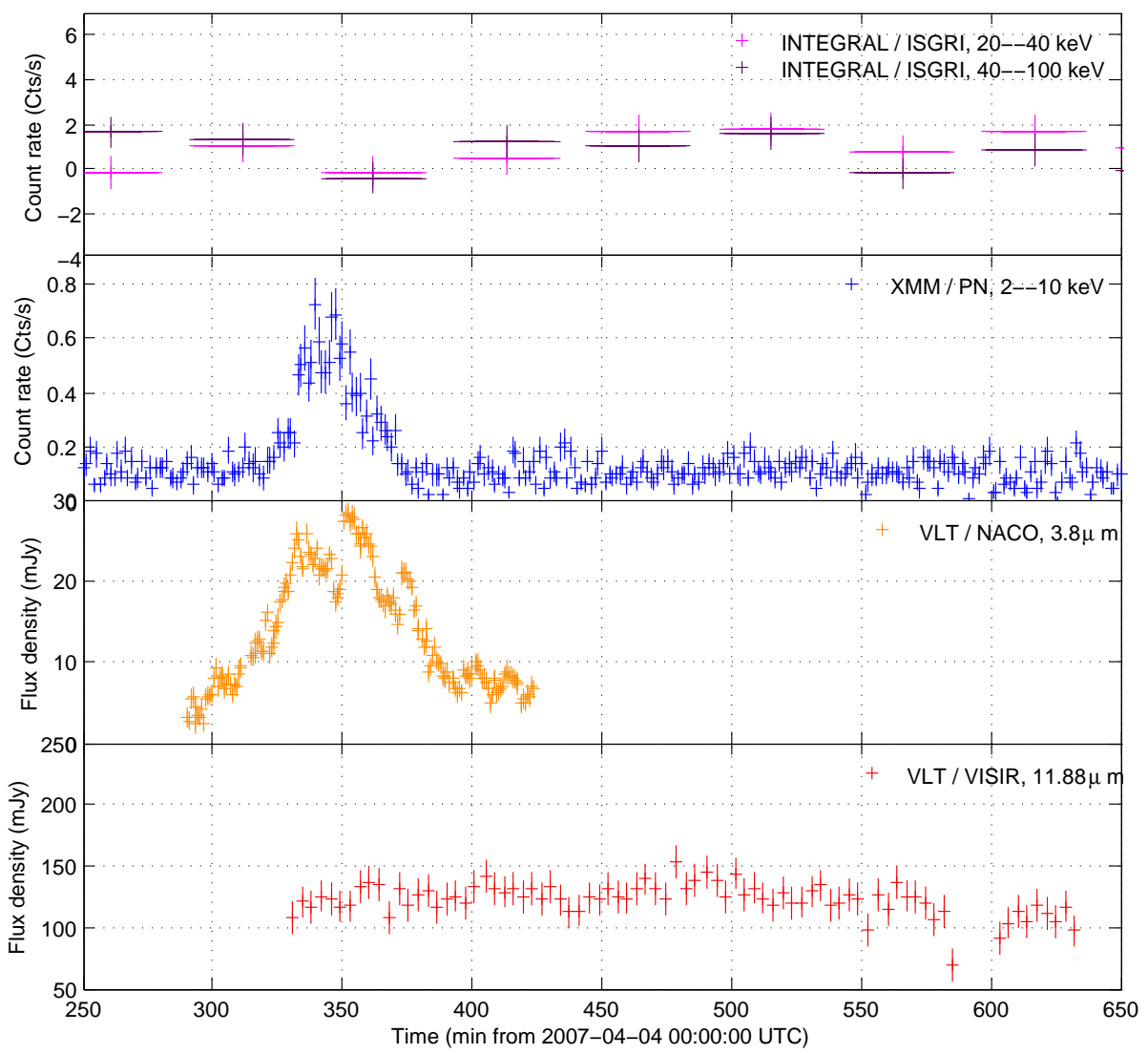

Fig. 4. From top to bottom, light curves of IGR J17456-2901 + AX J1745.6-2901, Sgr $A^{\star}+$ G 359.95-0.04 + IRS $13+$ diffuse emission, Sgr A* + S 17, and diffuse emission.

\subsection{Gamma-rays}

The INTEGRAL satellite (Winkler et al., 2003) monitored the GC in parallel to the other instruments in April 2007, for a total effective exposure of $\sim 212 \mathrm{ks}$ for IBIS/ISGRI (20-100 keV) (Ubertini et al., 2003; Lebrun et al., 2003 ) and $\sim 46$ ks for JEM-X 1 (3-20 keV) (Lund et al., 2003) ${ }^{11}$ at Sgr A* position. Measurements were spread over two consecutive revolutions, 545 and 546, from 2007-04-01 12:58:00 to 2007-04-02 21:32:34 and 2007-04-03 11:48:14 to 2007-04-04 20:26:59 (UT), respectively. In total, data of 74 individual pointings (science windows, ScWs) were acquired, lasting $\sim 2930$ s each. The whole

${ }^{11}$ Compared to IBIS, JEM-X field of view is narrower, so given the rectangular dithering pattern used $(5 \times 5$ pointings $=24$ off-source +1 on-source $)$, the GC was invisible to JEM-X most of the time, which explains the discrepancy in the exposures. 
dataset was reduced with OSA 7.0, the Offline Science Analysis Software, distributed by the INTEGRAL Science Data Center (ISDC) (Courvoisier et al., 2003), with algorithms described in Goldwurm et al. (2003b) for IBIS/ISGRI and Westergaard et al. (2003) for JEM-X.

To search for a counterpart above $20 \mathrm{keV}$ of the aforementioned flare, we selected the two consecutive ScWs of ISGRI that covered the flare time interval, 054600220010 and 054600230010, and created a combined mosaic of the individual images, in two energy bands: $20-40$ and 40-100 keV. None of these mosaics contained a distinctive source at the position of the black hole. Hence, no high energy counterpart of the flare was found. By considering the variance in Sgr A* pixel, we derive $3 \sigma$ upper limits on the flare of 1.17 and 1.11 cts s$^{-1}$ in the 20-40 and 40-100 keV bands, respectively. Assuming a power-law spectral shape of index $\Gamma=2.3$ (Sect. 2.1), these rates convert to flux limits of 5.76 and $11.1 \times 10^{-11} \mathrm{erg} \mathrm{s}^{-1} \mathrm{~cm}^{-2}$, respectively.

Regarding JEM-X 1 data in the $3-20 \mathrm{keV}$ band, we report similar results. There was no detection in the combined mosaic, and given a flare duration of $\sim 3000 \mathrm{~s}$, the sensitivity curves of JEM-X ${ }^{12}$ provide $5 \sigma$ upperlimits of 10 and $7 \times 10^{-11} \mathrm{erg} \mathrm{s}^{-1} \mathrm{~cm}^{-2}$ in the 3-10 and 10-25 keV energy ranges, respectively.

On Fig. 4 (top panel), we plotted the ISGRI light curves of the pixel at the position of Sgr $A^{\star}$, built with individual ScW. It is noteworthy that no source was significantly detected in any individual exposure.

In contrast to individual ScWs, the total ISGRI and JEM-X 1 mosaics of the observation dataset both reveal a significant excess at the position of Sgr $A^{\star}$ (see Fig. 1, top, left, and right). The significances of these signals are both $13.7 \sigma$. In view of the $X M M-N e w t o n$ image (Fig. 1, middle), the transient neutron star low-mass X-ray binary AX J1745.6-2901, located just 1.5' from $\mathrm{Sgr} \mathrm{A}^{\star}$ in projection, was markedly the dominant source of the region. Given JEM-X angular resolution of $\sim 3^{\prime}$ (FWHM), we can safely associate its 3-20 keV excess with the binary. In the ISGRI mosaic (Fig. 1, top, left), the PSF is $\sim 13^{\prime}$ (FWHM), and so does not allow us to disentangle AX J1745.62901 from IGR J17456-2901, the persistent hard X-ray source discovered by INTEGRAL/ISGR ${ }^{13}$ (Bélanger et al. 2004, 2006). To assess the contribution of the transient binary to the ISGRI signal though, we compared the April 2007 20-40 keV mosaic with another equivalent GC map, constructed with data spanning four months, from August to November 2006. During this latter period, we know for sure that the transient binary was in quiescence and undetected at high energies, thanks to a regular Swift/XRT monitoring of the GC

$\overline{12}$ See JEM-X user manual: http://isdc.unige.ch/Soft/download/osa/osa_doc/prod/osa_um_ibis-7.0.pdf ${ }^{13}$ Notice that IGR J17456-2901 has not been significantly detected with JEM-X yet. 
(Degenaar \& Wijnands, 2009). So, the total count rate of $0.86 \pm 0.03 \mathrm{cts} \mathrm{s}^{-1}$ we measured in the central pixel of the excess in the 2006 mosaic, can be entirely attributed to IGR J17456-2901. In April 2007, we found that the total count rate increased to $0.97 \pm 0.07 \mathrm{cts} \mathrm{s}^{-1}$, so that, presuming IGR J17456-2901 remained constant, the photons from the 20-40 keV excess visible in Fig. 1 (top, left) came at $\sim 90 \%$ from IGR J17456-2901 and 10\% from AX J1745.6-2901.

This is the first time INTEGRAL was gazing the GC during a period of known flaring activity from Sgr $A^{\star}$. Previous X-ray $/ \gamma$-ray coordinated campaigns in 2004 were, indeed, inconclusive, since the X-ray flares detected then by XMM-Newton occured at times when INTEGRAL was crossing the radiation belts with all its intruments in standby mode (Bélanger et al., 2006).

As indicated above, we did not identify any $\gamma$-ray counterpart of the intense X-ray flare from April $4^{\text {th }}$. This proves once again that Sgr A* does not release the bulk of its emission in soft $\gamma$-rays (Goldwurm et al., 1994). This result is also somewhat reminescent of the 2005 Chandra/HESS joint campaign, which demonstrated that the TeV source of the GC, HESS J1745-290, stayed still during an X-ray outburst seen by Chandra (Hinton et al., 2007a; Aharonian et al. 2008).

On Fig. 5, we display the broad band quiescent spectral energy distribution (SED) of Sgr $A^{\star}$ in dark gray. We also overplot in blue the spectral information on the 2007 April $4^{\text {th }}$ flare. By extrapolating the X-ray power-law, one expects fluxes of 3.9 and $4.1 \times 10^{-12} \mathrm{erg} \mathrm{s}^{-1} \mathrm{~cm}^{-2}$ in the $20-40$ and $40-100 \mathrm{keV}$, respectively. These expected values are roughly one order of magnitude below the $3 \sigma$ constraints worked out above, which suggest that the next generation of hard X-ray focusing instruments, like Simbol-X (Ferrando, 2008), will be able to extend spectral measurements on a flare of the GC supermassive black hole to above $20 \mathrm{keV}$ (Goldwurm, 2008).

Concerning IGR J17456-2901, it was relatively improbable to find it flare up in April 2007, based on the long ISGRI exposures targeted at the source in 2003 and 2004. These did not reveal any sign of variability on any timescale (Bélanger et al., 2006), despite a temporal artefact in the early light curves of IGR J17456-2901 (Bélanger et al., 2004), that was later attibuted to a poor correction of the background (Bélanger et al., 2006). Note, however, that variability on a single ScW duration basis cannot really be excluded, since this time interval is too short to convincingly detect the source IGR J17456-2901.

The provenance of IGR J17456-2901 thus remains enigmatic. We showed that the activity of the luminous transient binary AX J1745.6-2901 did not amount to more than $\sim 10 \%$ of the total $20-40 \mathrm{keV}$ flux of IGR J17456-2901, contrary to what was alluded to in Revnivtsev et al. (2004). The absence of variability and the fact that the flux of IGR J17456-2901 is two orders of 
magnitude above the quiescent emission of Sgr A* as measured by Chandra, supports the idea that the hard X-ray photons visible in INTEGRAL's mosaics are unlikely to be produced in the inner region of the accretion/ejection flow around the black hole. Instead, these photons should arise from a diffuse, and yet compact (a few arcminutes), zone, or maybe result from the sum of unresolved hard X-ray point sources (Revnivtsev et al., 2006). A possible connection between IGR J17456-2901 and HESS J1745-290 is another option. Hinton et al. (2007b) put forward that $\sim 10-100 \mathrm{TeV}$ electrons permeating the inner 20 pc may be responsible for the combined XMM-Newton/INTEGRAL spectrum of the central $8^{\prime}$ radius region (Bélanger et al. 2006) via synchrotron emission, as well as HESS J1745-290 through inverse Compton (IC) processes. These authors favor the pulsar wind nebula candidate G 359.95-0.04 (Wang et al., 2006) as the X-ray counterpart of HESS J1745-290, though. In their scenario, the $\mathrm{TeV}$ photons come about in the compact nebula, just $0.3 \mathrm{pc}$ from Sgr A*, by the IC boosting of ambient photons by relativistic electrons originating from the pulsar. Nevertheless, IGR J17456-2901 does not fit within this frame, as its flux is too high to be the simple hard X-ray extension of G 359.950.04 soft X-ray flux as determined by Chandra (Wang et al., 2006). The increased angular resolution and sensitivity in the hard X-ray range of the next generation of instruments will also help address the question of IGR J174562901 true nature.

\section{Radiative processes}

The extremely low quiescent luminosity of Sgr A* (10 orders of magnitude below the Eddington luminosity) is a long standing puzzle, that has stimulated numerous theoretical studies based on a combination of a low accretion rate, a radiatively inefficient accretion flow and outflows ejecting out the matter that just flowed in (See Melia \& Falcke, 2001, for a review and references therein). We will not discuss here the quiescent state and rather concentrate on the flaring emission, for which many models have also been proposed: jet models (Markoff et al., 2001), ADAF like models (Yuan et al., 2003, 2004) and accretion/stochastic acceleration models (Liu \& Melia, 2002; Liu et al., 2004, 2006a b). Each of them usually invokes either synchrotron self-Compton (SSC), external Compton (EC) or synchrotron broken power-laws (SB) processes as radiation mechanisms. In the subsequent discussion, we will explore these different possibilities for the April $4^{\text {th }}$ event, with no a priori assumption about the true nature of the engine behind the flare. We will examine the case of power-law distributions of electrons and highlight the natural synchrotron self-Compton component of each model. 


\subsection{Synchrotron self-Compton}

NIR flares are traditionally thought to arise from synchrotron emission since they are highly polarized (Eisenhauer et al., 2005; Gillessen et al., 2006; Krabbe et al., 2006; Hornstein et al., 2007) and have power-law spectral shapes (Eckart et al., 2006b; Meyer et al., 2006, 2007; Trippe et al., 2007). Here, we could not obtain a direct NIR spectrum of the flare, but we do have a stringent MIR upper limit. Hence, supposing a power-law spectral shape from MIR to NIR, $\nu F_{\nu}^{\mathrm{IR}} \propto \nu^{\beta_{\mathrm{IR}}}$, we have $\beta_{\mathrm{IR}}>0.04$. This is consistent with the index $\beta_{\mathrm{NIR}}=0.4$ published in previous NIR studies (Genzel et al., 2003; Gillessen et al., 2006, Hornstein et al., 2007). The submm bump of Sgr A* quiescent SED has a slope $\beta_{\text {submm }}^{\text {thick }}>0$ below $\sim 10^{12} \mathrm{~Hz}$, which is thought to arise from an optically thick regime, and a slope $\beta_{\text {submm }}^{\text {thin }}<0$ above $\sim 10^{12} \mathrm{~Hz}$, presumably coming from an optically thin regime, judging from polarization measurements (Aitken et al., 2000). The fact that $\beta_{\mathrm{IR}}>0$ around $10^{13} \mathrm{~Hz}$, shows that the NIR flare did not come from a global shift upward of the submm bump, but from a distinct population of particles creating a new rising hump in the SED in the IR band (see Fig. 5).

In an SSC model for the flares, the NIR photons are produced by a momentarily accelerated population of electrons radiating in the NIR band via a synchrotron process. In the following we will use the simple parametrization of Krawczynski et al. (2004) in which a spherical homogeneous source of synchrotron radiation with a radius $R$ and a volumic electron density $n_{\mathrm{e}}$, pervaded by a magnetic field $B$, has a power-law energy distribution:

$$
n(\gamma) \propto \gamma^{-p} \text { for } \quad \gamma_{\min }<\gamma<\gamma_{\max }
$$

We set $p=2$ in what follow: ${ }^{14}$. The electron density is thus determined by the normalization factor, $n_{0}$, of the power-law distribution by:

$$
n_{\mathrm{e}}=\int_{\gamma_{\min }}^{\gamma_{\max }} n(\gamma) d \gamma=\int_{\gamma_{\min }}^{\gamma_{\max }} n_{0} \gamma^{-2} d \gamma=-n_{0}\left(\gamma_{\max }^{-1}-\gamma_{\min }^{-1}\right)
$$

The energy density of the electrons, $w_{\mathrm{e}}$, used by Krawczynski et al. (2004) as normalization is also linked to $n_{0}$ through:

\footnotetext{
$\overline{{ }^{14} \text { Such }}$ an index could be the natural consequence of a Fermi II acceleration process for instance.
} 


$$
w_{\mathrm{e}}=\int_{\gamma_{\min }}^{\gamma_{\max }} \gamma m_{\mathrm{e}} c^{2} n(\gamma) d \gamma=n_{0} m_{\mathrm{e}} c^{2} \ln \frac{\gamma_{\max }}{\gamma_{\min }}
$$

The resulting synchrotron photon spectrum is optically thin and has a power-law shape (Rybicki \& Lightman, 1979):

$$
\nu F_{\nu}^{\mathrm{sync}} \propto n_{\mathrm{e}} R^{3} B^{(1+p) / 2} \nu^{(3-p) / 2} \quad \text { for } \quad \nu_{\min }^{\mathrm{sync}}<\nu<\nu_{\max }^{\mathrm{sync}}
$$

with $\nu_{\min (\max )}^{\mathrm{syc}} \propto \gamma_{\min (\max )}^{2} \nu_{g}$, where $\nu_{\mathrm{g}}=\frac{e B}{2 \pi m_{\mathrm{e}} c}$ is the gyration frequency and $m_{\mathrm{e}}$ the mass of the electron. Below $\nu_{\mathrm{min}}^{\mathrm{sync}}$, the photon spectrum has a powerlaw shape, $\nu F_{\nu}^{\text {sync }} \propto \nu^{\frac{4}{3}}$, due to the lowest energetic electrons which have a Lorentz factor $\gamma_{\min }$, and above $\nu_{\max }^{\text {sync }}$ it has an exponential cut-off.

In this SSC scheme, the X-ray flare is provoked by the inverse Compton boosting of the NIR flare photons by the same electrons that have just given rise to the NIR photons. The inverse Compton spectrum has the same morphology as the synchrotron one and scales like the Thomson optical depth of the sphere, $n_{\mathrm{e}} R \sigma_{\mathrm{T}}$, times $\nu F_{\nu}^{\text {sync: }}$

$$
\nu F_{\nu}^{\mathrm{ic}} \propto n_{\mathrm{e}} R \sigma_{\mathrm{T}} \times \nu F_{\nu}^{\mathrm{sync}} \propto n_{\mathrm{e}}^{2} R^{4} B^{(1+p) / 2} \nu^{(3-p) / 2} \quad \text { for } \quad \nu_{\min }^{\mathrm{ic}}<\nu<\nu_{\max }^{\mathrm{ic}},
$$

where $\sigma_{\mathrm{T}}$ stands for the Thomson cross-section ${ }^{15}$ and:

$$
\nu_{\min (\max )}^{\text {ic }} \propto \gamma_{\min (\max )}^{2} \nu_{\min (\max )}^{\mathrm{sync}} .
$$

Hence, this model has six free parameters: $p, \gamma_{\min }, \gamma_{\max }, B, R$, and $n_{\mathrm{e}}$. We arbitrarily fix $p=2, \gamma_{\min }=1$, and assess the four other parameters by considering four observables: the two frequencies of the synchrotron, $\nu_{\max }^{\text {sync }}$, and inverse Compton, $\nu_{\max }^{\text {ic }}$, peaks and their two respective amplitudes, $\nu F_{\nu}^{\text {sync,max }}$ and $\nu F_{\nu}^{\mathrm{ic}, \max }$. Indeed, on the one hand, $\gamma_{\max }$ is given by:

$$
\nu_{\max }^{\mathrm{ic}} / \nu_{\max }^{\mathrm{sync}} \simeq \gamma_{\max }^{2}
$$

and $B$ by (Rybicki \& Lightman, 1979) ${ }^{16}$,

$$
\nu_{\max }^{\text {sync }} \simeq 2.8\left(\frac{B}{1 \mathrm{G}}\right) \gamma_{\max }^{2} \times 10^{6} \mathrm{~Hz} .
$$

$\overline{{ }^{15} \sigma_{\mathrm{T}}=} \frac{8}{3} \pi r_{\mathrm{e}}^{2}$, where $r_{\mathrm{e}}=e^{2} /\left(m_{\mathrm{e}} c^{2}\right)=2.82 \times 10^{-13} \mathrm{~cm}$ is the classical radius of the electron.

16 This is an upper limit obtained for a pitch angle of $\pi / 2$. 
On the other hand, $R$ and $n_{\mathrm{e}}$ can be deduced from the relations:

$$
\left\{\begin{array}{l}
\frac{\nu F_{\nu}^{\mathrm{ic}, \max }}{\nu F_{\nu}^{\mathrm{sync}, \max }} \propto n_{\mathrm{e}} R \\
\nu F_{\nu}^{\text {sync,max }} \propto n_{\mathrm{e}} R^{3}
\end{array}\right.
$$

Yet, our measurements do not provide us these four observables per se. We know that the X-ray spectral slope is softer than the IR one, which suggests that our $X M M-N e w t o n$ measurement at $\nu_{\mathrm{X}} \approx 10^{18} \mathrm{~Hz}$ lies between the inverse Compton peak and the cut-off. Regarding the synchrotron peak, we only know it has a frequency $\nu_{\max }^{\text {sync }}>10^{14} \mathrm{~Hz}$. We will presume that this peak occurs at $\sim 10^{14} \mathrm{~Hz}$, in order to keep the magnetic field, $B$, as low as possible. As a result, we do not have to introduce the Klein-Nishina cross sections since the upscattering of the seed photons statisfies the Thomson regime condition, $\gamma_{\max } h \nu_{\text {seed }} \ll m_{\mathrm{e}} c^{2}$ (the transition to the Klein-Nishina regime is at $\sim 10^{18} \mathrm{~Hz}$ ).

With the set of parameters listed in Tab. 1 we obtain a good fit of the flare SED, as displayed in orange on Fig. 5. However the values of $B$ and $n_{\mathrm{e}}$ necessary to accommodate the data are extremely high. First, synchrotron cooling of the radiating particles has a characteristic timescale:

$$
\tau_{\text {cool }}=\left(\frac{1}{6 \pi} \frac{\sigma_{\mathrm{T}} B^{2}}{m_{\mathrm{e}} c} \gamma\left(1-\gamma^{-2}\right)\right)^{-1}=1.3\left(\frac{\nu}{1 \mathrm{~Hz}}\right)^{-\frac{1}{2}}\left(\frac{B}{1 \mathrm{G}}\right)^{-\frac{3}{2}} \times 10^{12} \mathrm{~s}
$$

This leads here, for such $B$, to $\tau_{\text {cool }} \approx 5 \mathrm{~s}$ at $\nu_{\mathrm{NIR}} \approx 10^{14} \mathrm{~Hz}$. Sustained injection of particles is therefore required to power the flare during 1-2 h. On top of that, the electron density is so high that synchrotron self-absorption (SSA) comes into play right below $8 \times 10^{13} \mathrm{~Hz}$, with an optically thick powerlaw $\nu F_{\nu}^{\mathrm{SSA}} \propto \nu^{\frac{7}{2}}$ (Rybicki \& Lightman, 1979). As a consequence, we cannot observe the power-law with index $(3-p) / 2$ for the synchrotron hump in contrast to the inverse Compton one, which also suffers from SSA but only at low frequencies. In any case the expected values for $B$ and $n_{\mathrm{e}}$ in the inner accretion flow around the black hole are orders of magnitude smaller $(B \approx 10 \mathrm{G}$ and $n_{\mathrm{e}} \approx 10^{7} \mathrm{~cm}^{-3}$ for Yuan et al. (2003)), even though interestingly the magnetic field at equipartition for particles with a typical energy $\gamma_{\max }=100$ and density $n_{\mathrm{e}}=2.2 \times 10^{12} \mathrm{~cm}^{-3}$ is $B_{\text {eq }} \simeq \sqrt{8 \pi \gamma_{\max } n_{\mathrm{e}} m_{\mathrm{e}} c^{2}} \approx 7 \times 10^{4} \mathrm{G}$, which is almost of the order of magnitude of the SSC magnetic field.

Another weakness of the model is that it predicts in sync variation for the NIR and X-ray light curves, i.e. there should also be visible substructure in the X-ray light curve. 
Table 1

Parameters of the radiative processes that match the SED of the April 2007 flare from Sgr A*.

\begin{tabular}{llll}
\hline Parameters & SSC & EC & SB \\
\hline \hline$p \ldots \ldots \ldots$ & 2 & 2 & $2-3$ \\
$\gamma_{\min } \ldots \ldots$ & 1 & 1 & 1 \\
$\gamma_{\max } \ldots \ldots$ & $10^{2}$ & $10^{3}$ & $9 \times 10^{4}$ \\
$\gamma_{\mathrm{br}} \ldots \ldots \ldots$ & - & - & $9 \times 10^{2}$ \\
$B(\mathrm{G}) \ldots \ldots$ & $10^{3}$ & 40 & 50 \\
$n_{\mathrm{e}}\left(\mathrm{cm}^{-3}\right)$. & $2.2 \times 10^{12}$ & $>1.8 \times 10^{10}$ & $7.6 \times 10^{6}$ \\
$R(\mathrm{~cm}) \ldots$ & $1.3 \times 10^{10}$ & $<1.6 \times 10^{11}$ & $1.4 \times 10^{12}$ \\
\hline
\end{tabular}

But an SSC scenario succeeds in explaining the simultaneity of the X-ray and NIR flares as well as the difference in their widths. Indeed, the SSC X-ray flux goes quadradically in $n_{\mathrm{e}}$ whereas the NIR flux goes linearly in $n_{\mathrm{e}}$. So, if one suppose the evolution of $n_{\mathrm{e}}(t)$ in time has a gaussian profile, then $n_{\mathrm{e}}(t)^{2}$ will have a width $1 / \sqrt{2}$ times narrower than $n_{\mathrm{e}}(t)$, which could explain the discrepancies of the X-ray and NIR light curves.

We expose here the results for a power-law energy distribution of electrons, but other distributions such as a relativistic Maxwellian (thermal distribution of typical Lorentz factor $\theta_{\mathrm{e}}=\frac{k T_{\mathrm{e}}}{m_{e} c^{2}}$ ) have been explored in past works (e.g. Liu et al., 2006b). Dodds-Eden et al. (2009) applied this distribution in an SSC pattern to this flare and found similar results for the physical parameters $B$, $R$, and $n_{\mathrm{e}}$. This is because both the power-law and the relativistic maxwellian distributions have a characteristic peak energy, $\gamma_{\max }$ or $\theta_{\mathrm{e}}$, which determines the relative positions of the synchrotron and inverse Compton bumps.

For the first time, we find it difficult to explain a simultaneous X-ray/NIR flare with SSC emission. As a matter of fact, past observations dealt with weaker flares and poorer spectral information. Eckart et al. (2006a) modeled their contemporaneous X-ray/NIR flares with SSC but had no individual Xray and NIR indices, so in particular the position of the inverse Compton peak was free, which relaxed the constraints on $B$. In contrast, Marrone et al. (2008) obtained individual NIR and X-ray spectra, though they were not exactly simultaneous. The flare was fainter than the one presented here and the $\mathrm{X}$-ray spectral index was consequently poorly constrained $\left(\beta_{\mathrm{X}}=0.0_{-1.0}^{+1.6}\right)$. The SED could thus be accommodated with a hard X-ray power-law, which again relaxed the constraints on $B$ and yielded acceptable SSC physical parameters. 


\subsection{External Compton}

Another alternative is that transiently accelerated relativistic electrons initiate the NIR flare through synchrotron and upscatter ambient low energy photons to the $\mathrm{keV}$ range, thus causing the X-ray flare. The most abundant source of photons around Sgr $\mathrm{A}^{\star}$ are the ones from the submm bump at $\nu_{\text {submm }} \approx 10^{12} \mathrm{~Hz}$ (see Fig. 5); we will designate by external Compton (EC) the comptonization of these photons by NIR electrons. We keep the same parametrization as for the SSC case in Sect. 3.1. We can estimate the maximal Lorentz factor of the particle and magnetic field as we did in the previous section, by switching respectively $\nu_{\max }^{\text {sync }}$ and $\nu_{\max }^{\text {ic }}$, by $\nu_{\text {submm }}$ and $\nu_{\mathrm{X}}$ in Eq. 11 and 2, By this means we find $\gamma_{\max } \approx 10^{3}$ and $B \approx 40 \mathrm{G}$. Such a magnetic field is more reasonable than in the SSC picture, the cooling time is $\sim 10 \mathrm{~min}$, and we no longer have to worry about SSA. Besides we know that whenever synchrotron and inverse Compton occur at the same place, the respective luminosities are linked via:

$$
\frac{L^{\text {ic }}}{L^{\text {sync }}}=\frac{U_{\text {seed }}}{U_{\mathrm{B}}}
$$

where $U_{\mathrm{B}}=\frac{B^{2}}{8 \pi}$ is the magnetic energy density and $U_{\text {seed }}$ is the seed photons energy density. If $A=4 \pi R_{\mathrm{Q}}^{2}$ denotes the surface area of the region of particles driving the quiescent submm lumninosity $L^{\mathrm{submm}}$, then $U_{\text {seed }}=\frac{L^{\mathrm{submm}}}{c A}$. Thereby, we can assess the radius of the quiescent emission:

$$
R_{\mathrm{Q}} \simeq 0.016 \times 10^{12}\left(\frac{L_{\mathrm{NIR}}}{L_{\odot}}\right)^{\frac{1}{2}}\left(\frac{L_{\mathrm{submm}}}{L_{\odot}}\right)^{\frac{1}{2}}\left(\frac{L_{\mathrm{X}}}{L_{\odot}}\right)^{-\frac{1}{2}}\left(\frac{B}{40 \mathrm{G}}\right)^{-1} \mathrm{~cm}
$$

where the solar luminosity is $L_{\odot}=3.8 \times 10^{33} \mathrm{erg} \mathrm{s}^{-1}$. The resulting radius is $R_{\mathrm{Q}} \approx 0.1 R_{\mathrm{S}}$, and this is probably the main weakness of the EC scenario, because VLBI measurements of $\mathrm{Sgr} \mathrm{A}^{\star}$ at $1.3 \mathrm{~mm}$ give an intrinsic size for the quiescent region of the order of the Schwarzschild radius (Doeleman et al., 2008). To compute the electron density $n_{\mathrm{e}}$ and the size of the flaring region $R$, a detailed treatment of the quiescence spectrum has to be taken into account, as done by Dodds-Eden et al. (2009) with the quiescent RIAF model of Yuan et al. (2003). Just to get a feeling of these parameters, we will consider that the flare is embedded in the submm region so that $R<R_{\mathrm{Q}}$ and consequently we find that $n_{\mathrm{e}}$ must be at least $1.8 \times 10^{10} \mathrm{~cm}^{-3}$ to fit the NIR synchrotron data.

To complement the study of Dodds-Eden et al. (2009), we have computed 
the synchrotron self-Compton emission that will naturally come along in the EC scheme. Interestingly, this contribution peaks in the soft gamma-ray band, less than one order of magnitude below the INTEGRAL upper limits (see Fig. 5, dashed line). If this scenario is real, it would tend to flatten the X-ray spectral slope towards high energies, thus making the prospects for future soft gamma-ray missions promising.

As in the SSC scheme, EC ensures simultaneity of the flare in the X-ray and NIR light curves. It could also provide an explanation for the absence of substructures in the X-ray light curve. Indeed, if one naively neglect the synchrotron losses, then the synchrotron luminosity is proportional to $B^{\frac{3}{2}}$ whereas the inverse Compton luminosity is, a priori, independent of $B$ because it depends on $L_{\text {submm }}$ and not on $L_{\mathrm{NIR}}$ as for SSC. So the NIR flare should be subject to $B$ variations contrary to the X-ray flare. A clumpy magnetic field in which the flaring region moves is maybe the key to these observed or unobserved features.

Yusef-Zadeh et al. (2006a) proposed that their synchronous observation of a flare with the HST and XMM-Newton in 2004 resulted from EC with an acceptable size of the submm quiescence region of $\sim 10 R_{\mathrm{S}}$. This was possible only because the spectrum of the $\mathrm{X}$-ray flare was hard, $\beta_{\mathrm{X}} \approx 0.5$ (Bélanger et al. 2005), and therefore allowed for a larger $\gamma_{\max }$, a lower $B$ and larger $R_{\mathrm{Q}}$. These authors also pointed out that the quiescent electrons reponsible for the submm bump were also likely to upscatter the NIR flare photons to keV energies as well, even though this may be a second order effect (Dodds-Eden et al. 2009).

\subsection{Synchrotron with break}

From our flare observations, it is clear that $\beta_{\mathrm{NIR}} \neq \beta_{\mathrm{X}}$ so that we cannot fit the entire SED of the flare with a single synchrotron power-law. But broad synchrotron power-laws are known to exhibit breaks of several kinds. In particular, a natural break comes from the synchrotron cooling of the electrons, which generates a difference of slopes of $|\Delta p|=1$ in the electrons distribution (and $|\Delta \beta|=0.5$ in the photons distribution) between the power-law below and above the break. This simple "synchrotron with a break" model (SB) would actually suit our measurements. The frequency at which the break is supposed to occur depends upon the modeling adopted. Here, we assume a "leaking box" model in which a constant injection of fresh particles is balanced by the synchrotron cooling of this electrons on a timescale $\tau_{\text {cool }}$ and their escape on another typical timescale. For the latter variable, we will take the the

dynamical timescale $\tau_{\text {dyn }}=\sqrt{\frac{r^{3}}{2 G M_{\bullet}}} \approx 5 \mathrm{~min}$, where $r=3 R_{\mathrm{S}}$ is the radius of the last stable orbit for a non spinning black hole. The condition $\tau_{\text {cool }}=\tau_{\text {dyn }}$ 


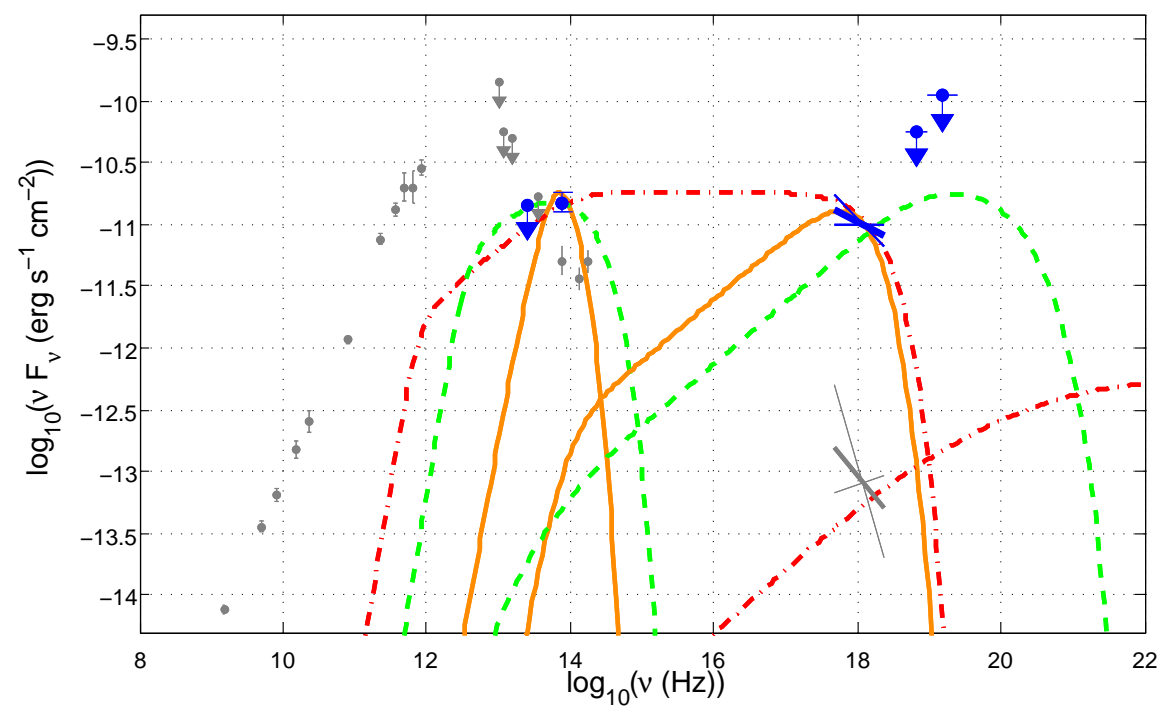

Fig. 5. Spectral energy distribution of Sgr A^. Dark gray measurements correspond to the quiescent state. Radio and submm points are extracted from Zhao et al. (2001); Zylka et al. (1995); Marrone et al. (2008), FIR and MIR upperlimits from Telesco et al. (1996); Cotera et al. (1999); Eckart et al. (2006a); Schödel et al. (2007), NIR points from Genzel et al. (2003) and the X-ray bow tie from Baganoff et al. (2003a). The XMM-Newton/EPIC spectrum of the April $4^{\text {th }}$ flare, the mean $V L T / \mathrm{NACO}$ flux, and the VLT/VISIR and INTEGRAL/ISGRI upper limits are overplotted in blue. The synchrotron and synchrotron self-Compton contributions of the SSC, EC, and SB models are plotted in orange (solid lines), green (dashed lines), and red (dash dotted lines), respectively (see the parameters of the fit in Tab. 11).

provides the frequency of the spectral break:

$$
\nu_{\mathrm{br}}=6.37\left(\frac{B}{1 \mathrm{G}}\right)^{-3} \times 10^{18} \mathrm{~Hz} .
$$

In Tab. 1 we list a sketch of physical parameters for SB that match the SED well. Again we chose $\gamma_{\min }=1$, and $\gamma_{\max }=9 \times 10^{4}$ to engender photons up to the X-ray range. $B$ was chosen to satisfy Eq. 3 for a break at $\sim 10^{14} \mathrm{~Hz}$. Finally, $n_{\mathrm{e}}$ and $R$ were adjusted to normalize the spectrum with reasonable values and in order not to violate our $\gamma$-ray constraints with the natural SSC component (see the rising SSC hump in red on bottom right of Fig. 5) coming along with synchrotron radiation. Note once more on Fig. 5 the SSA below $\sim 10^{12} \mathrm{~Hz}$.

SB is appealing because, compared to SSC and EC, it yields less extreme values in terms of $B, n_{\mathrm{e}}$, and $R$. However, in the SB case, there is no obvious justification for the differents durations of the flare in X-rays and NIR, and the presence/absence of substructures in the light curves. Here, we only discuss the average spectrum of the flare, where SB certainly requires a more detailed 
examination of the time evolution of the phenomenon, beyond the scope of this paper.

\section{Conclusions}

This paper complements a series of articles about the April 2007 synchronous observations of the Galactic Center from radio to $\gamma$-rays (Porquet et al., 2008; Dodds-Eden et al., 2009, Yusef-Zadeh et al., 2009). Here, we have recapped the results on the brightest flare ever detected simultaneously at NIR and X-ray frequencies. We have also reported for the first time $\gamma$-ray constraints on such an event, which, added to our MIR/NIR/X-ray spectral measurements, constitute the broadest simulaneous spectrum of a flare ever achieved. The essential observational conclusions may be summarized as follows:

- the peaks of the X-ray and NIR emissions are coincident within 3 min;

- the width of the NIR flare light curve is broader than the X-ray one by a factor $\sim 2$;

- the NIR light curve is substructured on a timescale of $\sim 20$ min while the $\mathrm{X}$-ray light curve is rather smooth;

- there is no detectable MIR counterpart;

- the soft $\gamma$-ray source IGR J17456-2901 is non variable.

The high quality of the spectral information we gathered allowed for a discussion of the several classical radiative processes models employed to explain the flares: SSC, EC, and SB. Yet, none of these mechanisms is entirely satisfactory to meet our observations. The theoretical inquiries to come will have to take into account the time evolution of the phenomenon and the aging of the radiating particles to better connect the light curves and spectra. From an observational stand point, it will be useful to repeat such NIR/X-ray measurements in a near future to get two respective individual and fully contemporaneous spectra, which has never been accomplished thus far. As we have seen, one key probe of what powers the flares, is a better determination of the X-ray spectral slope. In a more distant future, thanks to a broad X-ray sensitivity over the 1-80 keV band and a high angular resolution above $10 \mathrm{keV}$, Simbol-X should address this issue and resolve the GC region in soft $\gamma$-rays.

\section{Acknowledgements}

GT acknowledges M. Falanga, D. Götz, and J. Chenevez for help with the INTEGRAL data analysis, Y. Clénet, B. Draine, and M. Morris for useful dis- 
cussions about NIR/MIR measurements, and CEA Saclay for financial support to attend the $37^{\text {th }}$ COSPAR meeting in Montreal.

Part of this work has been funded by the french Agence Nationale pour la Recherche through grant ANR-06-JCJC-0047. At Arizona, this work was also supported by NASA grants NNX08AX33G and NNX08AX34G.

INTEGRAL is an ESA project with instruments and science data center funded by ESA member states (especially the PI countries: Denmark, France, Germany, Italy, Switzerland, and Spain), the Czech Republic, and Poland, and with the participation of Russia and the US.

The XMM-Newton project is an ESA Science Mission with instruments and contributions directly funded by ESA Member States and the USA (NASA).

\section{References}

Aharonian, F., Akhperjanian, A.G., Barres de Almeida, U., Bazer-Bachi, A.R., Becherini, Y., Behera, B., Benbow, W., Bernlöhr, K., Boisson, C., Bochow, A., Borrel, V., Braun, I., Brion, E., Brucker, J., Brun, P., Bühler, R., Bulik, T., Büsching, I., Boutelier, T., Carrigan, S., Chadwick, P.M., Charbonnier, A., Chaves, R.C.G., Cheesebrough, A., Chounet, L.-M., Clapson, A.C., Coignet, G., Dalton, M., Degrange, B., Deil, C., Dickinson, H.J., DjannatiAtaï, A., Domainko, W., O’C.Drury, L., Dubois, F., Dubus, G., Dyks, J., Dyrda, M., Egberts, K., Emmanoulopoulos, D., Espigat, P., Farnier, C., Feinstein, F., Fiasson, A., Förster, A., Fontaine, G., Füßling, M., Gabici, S., Gallant, Y.A., Gérard, L., Giebels, B., Glicenstein, J.F., Glück, B., Goret, P., Hadjichristidis, C., Hauser, D., Hauser, M., Heinz, S., Heinzelmann, G., Henri, G., Hermann, G., Hinton, J.A., Hoffmann, A., Hofmann, W., Holleran, M., Hoppe, S., Horns, D., Jacholkowska, A., de Jager, O.C., Jung, I., Katarzyński, K., Kaufmann, S., Kendziorra, E., Kerschhaggl, M., Khangulyan, D., Khélifi, B., Keogh, D., Komin, N., Kosack, K., Lamanna, G., Lenain, J.-P., Lohse, T., Marandon, V., Martin, J.M., Martineau-Huynh, O., Marcowith, A., Maurin, D., McComb, T.J.L., Medina, M.C., Moderski, R., Moulin, E., Naumann-Godo, M., de Naurois, M., Nedbal, D., Nekrassov, D., Niemiec, J., Nolan, S.J., Ohm, S., Olive, J.-F., de Ona Wilhelmi, E., Orford, K.J., Osborne, J.L., Ostrowski, M., Panter, M., Pedaletti, G., Pelletier, G., Petrucci, P.-O., Pita, S., Pühlhofer, G., Punch, M., Quirrenbach, A., Raubenheimer, B.C., Raue, M., Rayner, S.M., Renaud, M., Rieger, F., Ripken, J., Rob, L., Rosier-Lees, S., Rowell, G., Rudak, B., Rulten, C.B., Ruppel, J., Sahakian, V., Santangelo, A., Schlickeiser, R., Schöck, F.M., Schröder, R., Schwanke, U., Schwarzburg, S., Schwemmer, S., Shalchi, A., Skilton, J.L., Sol, H., Spangler, D., Stawarz, Ł., Steenkamp, R., Stegmann, C., Superina, G., Tam, P.H., Tavernet, J.-P., Terrier, R., Tibolla, O., van 
Eldik, C., Vasileiadis, G., Venter, C., Vialle, J.P., Vincent, P., Vivier, M., Völk, H.J., Volpe, F., Wagner, S.J., Ward, M., Zdziarski, A.A. \& Zech, A., Simultaneous HESS and Chandra observations of Sagitarius A* during an X-ray flare, A\&A, 492, L25-L28, 2008.

Aitken, D.K., Greaves, J., Chrysostomou, A., Jenness, T., Holland, W., Hough, J.H., Pierce-Price, D., Richer, J., Detection of Polarized Millimeter and Submillimeter Emission from Sagittarius A*, ApJ, 534, L173-L176, 2000.

Baganoff, F.K., Bautz, M.W., Brandt, W.N., Chartas, G., Feigelson, E.D., Garmire, G.P., Maeda, Y., Morris, M., Ricker, G.R., Townsley, L.K. \& Walter, F., Rapid X-ray flaring from the direction of the supermassive black hole at the Galactic Centre, Nature, 413, 45-48, 2001.

Baganoff, F.K., Maeda, Y., Morris, M., Bautz, M.W., Brandt, W.N., Cui, W., Doty, J.P., Feigelson, E.D., Garmire, G.P., Pravdo, S.H., Ricker, G.R. \& Townsley, L.K., Chandra X-Ray Spectroscopic Imaging of Sagittarius A* and the Central Parsec of the Galaxy, Nature, 591, 891-915, 2003a.

Baganoff, F.K., Bautz, M.W., Ricker, G.R., Morris, M., Becklin, E.E., Ghez, A.M., Hornstein, S.D., Tanner, A.M., Brandt, W.N., Chartas, G., Feigelson, E.D., Garmire, G.P., Cotera, A.S., Hinz, P.M., Hoffmann, W.F., Meyer, M.R., Eckart, A., Genzel, R., Zhao, J.-H., Herrnstein, R.M., Hora, J.L., Macquart, J.-P., Maeda, Y., Sault, R.J., Taylor, G.B. \& Walter, F., Multiwavelength Monitoring of Sgr A* During Chandra Observations of Multiple X-ray Flares, BAAS, 34, 1153, 2003b.

Balick, B. \& Brown, R.L., Intense sub-arcsecond structure in the galactic center, ApJ, 194, 265-270, 1974.

Bélanger, G., Goldwurm, A., Goldoni, P., Paul, J., Terrier, R., Falanga, M., Ubertini, P., Bazzano, A., Del Santo, M., Winkler, C., Parmar, A.N., Kuulkers, E., Ebisawa, K., Roques, J.P., Lund, N., \& Melia, F., Detection of Hard X-Ray Emission from the Galactic Nuclear Region with INTEGRAL, ApJ, 601, L163-L166, 2004.

Bélanger, G., Goldwurm, A., Melia, F., Ferrando, P., Grosso, N., Porquet, D., Warwick, R. \& Yusef-Zadeh, F., Repeated X-ray Flaring Activity in Sagittarius A*, ApJ, 635, 1095-1102, 2005.

Bélanger, G., Goldwurm, A., Renaud, M., Terrier, R., Melia, F., Lund, N., Paul, J., Skinner, G. \& Yusef-Zadeh, F., A Persistent High-energy Flux from the Heart of the Milky Way: INTEGRAL's View of the Galactic Center, ApJ, 636, 275-289, 2006.

Čadež, A., Calvani, M. \& Kostić, U., On the tidal evolution of the orbits of low-mass satellites around black holes, A\&A, 487, 527-532, 2008.

Chiar, J.E. \& Tielens, A.G.G.M., A Persistent High-energy Flux from the Heart of the Milky Way: INTEGRAL's View of the Galactic Center, ApJ, 637, 774-785, 2006.

Clénet, Y., Rouan, D., Gratadour, D., Marco, O., Léna, P., Ageorges, N. \& Gendron, E., A dual emission mechanism in Sgr A*/L'?, A\&A, 439, L9-L13, 2005.

Cohen, M., Walker, R.G., Carter, B., Hammersley, P., Kidger, M. \& Noguchi, 
K., Spectral Irradiance Calibration in the Infrared. X. A Self-Consistent Radiometric All-Sky Network of Absolutely Calibrated Stellar Spectra, AJ, 117, 1864-1889, 1999.

Cotera, A., Morris, M., Ghez, A.M., Becklin, E.E., Tanner, A.M., Werner, M.W. \& Stolovy, S.R., Mid-Infrared Imaging of the Central Parsec with Keck, ASPC, 186, 240, 1999.

Courvoisier, T.J.-L., Walter, R., Beckmann, V., Dean, A.J., Dubath, P., Hudec, R., Kretschmar, P., Mereghetti, S., Montmerle, T., Mowlavi, N., Paltani, S., Preite Martinez, A., Produit, N., Staubert, R., Strong, A.W., Swings, J.-P., Westergaard, N.J., White, N., Winkler, C. \& Zdziarski, A.A., The INTEGRAL Science Data Centre (ISDC), A\&A, 411, L53-L57, 2003.

Degenaar, N. \& Wijnands, R., The behavior of subluminous X-ray transients near the Galactic center as observed using the X-ray telescope aboard Swift, A\&A, 495, 547-559, 2009.

Do, T., Ghez, A.M., Morris, M.R., Yelda, S., Meyer, L., Lu, J.R., Hornstein, S.D. \& Matthews, K., A Near-Infrared Variability Study of the Galactic Black Hole: A Red Noise Source with NO Detected Periodicity, ApJ, 691, 1021-1034, 2009.

Dodds-Eden, K., Porquet, D., Trap, G., Quataert, E., Haubois, X., Gillessen, S., Grosso, N., Pantin, E., Falcke, H., Rouan, D., Genzel, R., Hasinger, G., Goldwurm, A., Yusef-Zadeh, F., Clenet, Y., Trippe, S., Lagage, P.-O., Bartko, H., Eisenhauer, F., Ott, T., Paumard, T., Perrin, G., Yuan, F., Fritz, T.K. \& Mascetti, L., Evidence for X-ray synchrotron emission from simultaneous mid-IR to X-ray observations of a strong Sgr A* flare, ApJ, 698, 676-692, 2009.

Doeleman, S.S., Weintroub, J., Rogers, A.E.E., Plambeck, R., Freund, R., Tilanus, R.P.J., Friberg, P., Ziurys, L.M., Moran, J.M., Corey, B., Young, K.H., Smythe, D.L., Titus, M., Marrone, D.P., Cappallo, R.J., Bock, D.C.J., Bower, G.C., Chamberlin, R., Davis, G.R., Krichbaum, T.P., Lamb, J., Maness, H., Niell, A.E., Roy, A., Strittmatter, P., Werthimer, D., Whitney, A.R. \& Woody, D., Event-horizon-scale structure in the supermassive black hole candidate at the Galactic Centre, Nature, 455, 78-80, 2008.

Eckart, A., Baganoff, F.K., Morris, M., Bautz, M.W., Brandt, W.N., Garmire, G.P., Genzel, R., Ott, T., Ricker, G.R., Straubmeier, C., Viehmann, T., Schödel, R., Bower, G.C. \& Goldston, J.E., First simultaneous NIR/X-ray detection of a flare from Sgr A*, A\&A, 427, 1-11, 2004.

Eckart, A., Baganoff, F.K., Schödel, R., Morris, M., Genzel, R., Bower, G.C., Marrone, D., Moran, J.M., Viehmann, T., Bautz, M.W., Brandt, W.N., Garmire, G.P., Ott, T., Trippe, S., Ricker, G.R., Straubmeier, C., Roberts, D.A., Yusef-Zadeh, F., Zhao, J.H. \& Rao, R., The flare activity of Sagittarius A*. New coordinated mm to X-ray observations, A\&A, 450, 535-555, 2006.

Eckart, A., Schödel, R., Meyer, L., Trippe, S., Ott, T. \& Genzel, R., Polarimetry of near-infrared flares from Sagittarius A*, A\&A, 455, 1-10, 2006.

Eckart, A., Baganoff, F.K., Zamaninasab, M., Morris, M.R., Schödel, R., Meyer, L., Muzic, K., Bautz, M.W., Brandt, W.N., Garmire, G.P., Ricker, 
G.R., Kunneriath, D., Straubmeier, C., Duschl, W., Dovciak, M., Karas, V., Markoff, S., Najarro, F., Mauerhan, J., Moultaka, J. \& Zensus, A., Polarized NIR and X-ray flares from Sagittarius A*, A\&A, 479, 625-639, 2008a.

Eckart, A., Schödel, R., García-Marín, M., Witzel, G., Weiss, A., Baganoff, F.K., Morris, M.R., Bertram, T., Dovčiak, M., Duschl, W.J., Karas, V., König, S., Krichbaum, T.P., Krips, M., Kunneriath, D., Lu, R.-S., Markoff, S., Mauerhan, J., Meyer, L., Moultaka, J., Mužić, K., Najarro, F., Pott, J.-U., Schuster, K.F., Sjouwerman, L.O., Straubmeier, C., Thum, C., Vogel, S.N., Wiesemeyer, H., Zamaninasab, M. \& Zensus, J.A., Simultaneous NIR/sub-mm observation of flare emission from SgrA*, A\&A, 492, 337-344, 2008b.

Eisenhauer, F., Genzel, R., Alexander, T., Abuter, R., Paumard, T., Ott, T., Gilbert, A., Gillessen, S., Horrobin, M., Trippe, S., Bonnet, H., Dumas, C., Hubin, N., Kaufer, A., Kissler-Patig, M., Monnet, G., Ströbele, S., Szeifert, T., Eckart, A., Schödel, R. \& Zucker, S., SINFONI in the Galactic Center: Young Stars and Infrared Flares in the Central Light-Month, ApJ, 628, 246-259, 2005.

Falanga, M. and Melia, F. and Tagger, M. and Goldwurm, A. and Bélanger, G., General Relativistic Flux Modulations from Disk Instabilities in Sagittarius A*, ApJ, 662, L15-L18, 2007.

Falanga, M. and Melia, F. and Prescher, M. and Bélanger, G. and Goldwurm, A.., Modulated X-Ray Emissivity near the Stress Edge in Sagittarius A*, ApJ, 679, L93-L96, 2008.

Ferrando, P., The Simbol-X mission, Memorie della Societa Astronomica Italiana, 79, 19, 2008.

Genzel, R., Schödel, R., Ott, T., Eckart, A., Alexander, T., Lacombe, F., Rouan, D. \& Aschenbach, B., Near-infrared flares from accreting gas around the supermassive black hole at the Galactic Centre, Nature, 425, 934-937, 2003.

Ghez, A.M., Duchêne, G., Matthews, K., Hornstein, S.D., Tanner, A., Larkin, J., Morris, M., Becklin, E.E., Salim, S., Kremenek, T., Thompson, D., Soifer, B.T., Neugebauer, G. \& McLean, I., The First Measurement of Spectral Lines in a Short-Period Star Bound to the Galaxy's Central Black Hole: A Paradox of Youth, ApJ, 586, L127-L131, 2003

Ghez, A.M., Wright, S.A., Matthews, K., Thompson, D., Le Mignant, D., Tanner, A., Hornstein, S.D., Morris, M., Becklin, E.E. \& Soifer, B.T., Variable Infrared Emission from the Supermassive Black Hole at the Center of the Milky Way, ApJ, 601, L159-L162, 2004

Ghez, A.M., Hornstein, S.D., Lu, J.R., Bouchez, A., Le Mignant, D., van Dam, M.A., Wizinowich, P., Matthews, K., Morris, M., Becklin, E.E., Campbell, R.D., Chin, J.C.Y., Hartman, S.K., Johansson, E.M., Lafon, R.E., Stomski, P.J. \& Summers, D.M., The First Laser Guide Star Adaptive Optics Observations of the Galactic Center: Sgr A*'s Infrared Color and the Extended Red Emission in its Vicinity, ApJ, 635, 1087-1094, 2005

Ghez, A.M., Salim, S., Weinberg, N.N., Lu, J.R., Do, T., Dunn, J.K., 
Matthews, K., Morris, M., Yelda, S., Becklin, E.E., Kremenek, T., Milosavljevic, M. \& Naiman, J., Measuring Distance and Properties of the Milky Way's Central Supermassive Black Hole with Stellar Orbits, ApJ, 689, 10441062, 2008

Gillessen, S., Eisenhauer, F., Quataert, E., Genzel, R., Paumard, T., Trippe, S., Ott, T., Abuter, R., Eckart, A., Lagage, P.O., Lehnert, M.D., Tacconi, L.J. \& Martins, F., Variations in the Spectral Slope of Sagittarius A* during a Near-Infrared Flare, ApJ, 640, L163-L166, 2006

Goldwurm, A., Cordier, B., Paul, J., Ballet, J., Bouchet, L., Roques, J.P., Vedrenne, G., Mandroui, P., Sunyaev, R., Churazov, E., Gilfanov, M., Finogenov, A., Vikhlinin, A., Dyachkov, A., Khavenson, N. \& Kovtunenko, V., Evidence against a Massive Black-Hole at the Galactic Center, Nature, 371, 589, 1994.

Goldwurm, A., Observing the Super-Massive Black Hole of the Galactic center with Simbol-X, Memorie della Societa Astronomica Italiana, 79, 81, 2008.

Goldwurm, A., Brion, E., Goldoni, P., Ferrando, P., Daigne, F., Decourchelle, A., Warwick, R.S. \& Predehl, P., A New X-Ray Flare from the Galactic Nucleus Detected with the XMM-Newton Photon Imaging Cameras, A\&A, 584, 751-757, 2003a.

Goldwurm, A., David, P., Foschini, L., Gros, A., Laurent, P., Sauvageon, A., Bird, A.J., Lerusse, L. \& Produit, N., The INTEGRAL/IBIS scientific data analysis, A\&A, 411, L223-L229, 2003b.

Grosso, N., Porquet, D. \& Boirin, L., XMM-Newton view of the eclipsing burster low-mass X-ray binary AX J1745.6-2901, in Proceedings of the SF2A, 233, 2008.

Haubois, X. , Genzel, R. , Perrin, G. , Gillessen, S. , Paumard, T. , DoddsEden, K. , Clénet, Y. \& Rouan, D., The Galactic Center large program, AIPCS, 1053, 91-94, 2008.

Herrnstein, R.M., Zhao, J.-H., Bower, G.C. \& Goss, W.M., The Variability of Sagittarius A* at Centimeter Wavelengths, AJ, 127, 3399-3410, 2004.

Hinton, J., Vivier, M., Bühler, R., Pühlhofer, G. \& Wagner, S., Simultaneous H.E.S.S. and Chandra observations of Sgr A* during an X-ray flare, ICRC, arXiv:0710.1537, 2007a.

Hinton, J.A. \& Aharonian, F.A., Inverse Compton Scenarios for the TeV Gamma-Ray Emission of the Galactic Center, ApJ 657, 302-307, 2007b.

Hornstein, S.D., Matthews, K., Ghez, A.M., Lu, J.R., Morris, M., Becklin, E.E., Rafelski, M. \& Baganoff, F.K., A Constant Spectral Index for Sagittarius A* during Infrared/X-Ray Intensity Variations, ApJ, 667, 900-910, 2007.

Jansen, F., Lumb, D., Altieri, B., Clavel, J., Ehle, M., Erd, C., Gabriel, C., Guainazzi, M., Gondoin, P., Much, R., Munoz, R., Santos, M., Schartel, N., Texier, D. \& Vacanti, G., XMM-Newton observatory. I. The spacecraft and operations, A\&A, 365, L1-L6, 2001.

Krabbe, A., Iserlohe, C., Larkin, J.E., Barczys, M., McElwain, M., Weiss, J., Wright, S.A. \& Quirrenbach, A., Diffraction-limited Imaging Spectroscopy 
of the Sagittarius A* Region Using OSIRIS, a New Keck Instrument, ApJ, 642, L145-L148, 2006.

Krawczynski, H., Hughes, S.B., Horan, D., Aharonian, F., Aller, M.F., Aller, H., Boltwood, P., Buckley, J., Coppi, P., Fossati, G., Götting, N., Holder, J., Horns, D., Kurtanidze, O.M., Marscher, A.P., Nikolashvili, M., Remillard, R.A., Sadun, A. \& Schröder, M., Multiwavelength Observations of Strong Flares from the TeV Blazar 1ES 1959+650, ApJ, 601, 151-164, 2004.

Lagage, P.O., Pel, J.W., Authier, M., Belorgey, J., Claret, A., Doucet, C., Dubreuil, D., Durand, G., Elswijk, E., Girardot, P., Käufl, H.U., Kroes, G., Lortholary, M., Lussignol, Y., Marchesi, M., Pantin, E., Peletier, R., Pirard, J.-F., Pragt, J., Rio, Y., Schoenmaker, T., Siebenmorgen, R., Silber, A., Smette, A., Sterzik, M. \& Veyssiere, C., Successful Commissioning of VISIR: The Mid-Infrared VLT Instrument, The Messenger, 117, 12-16, 2004.

Lebrun, F., Leray, J.P., Lavocat, P., Crétolle, J., Arquès, M., Blondel, C., Bonnin, C., Bouère, A., Cara, C., Chaleil, T., Daly, F., Desages, F., Dzitko, H., Horeau, B., Laurent, P., Limousin, O., Mathy, F., Mauguen, V., Meignier, F., Molinié, F., Poindron, E., Rouger, M., Sauvageon, A. \& Tourrette, T., ISGRI: The INTEGRAL Soft Gamma-Ray Imager, A\&A, 411, L141-L148, 2003.

Lenzen, R., Hartung, M., Brandner, W., Finger, G., Hubin, N.N., Lacombe, F., Lagrange, A.-M., Lehnert, M.D., Moorwood, A.F.M. \& Mouillet, D., NAOS-CONICA first on sky results in a variety of observing modes, SPIE, 4841, 944-952, 2003.

Liu, S. \& Melia, F., An Accretion-induced X-Ray Flare in Sagittarius A*, ApJ, 566, 77-80, 2002.

Liu, S., Petrosian, V. \& Melia, F., A Testable Stochastic Acceleration Model for Flares in Sagittarius A*, ApJ, 611, L101-L104, 2004.

Liu, S. and Melia, F. \& Petrosian, V., Stochastic electron acceleration during the near-infrared and X-ray flares in Sagittarius A*, ApJ, 636, 798-803, 2006a.

Liu, S., Petrosian, V., Melia, F. \& Fryer, C.L., A Testable Stochastic Acceleration Model for Flares in Sagittarius A*, ApJ, 648, 1020-1025, 2006b.

Lund, N., Budtz-Jørgensen, C., Westergaard, N.J., Brandt, S., Rasmussen, I.L., Hornstrup, A., Oxborrow, C.A., Chenevez, J., Jensen, P.A., Laursen, S., Andersen, K.H., Mogensen, P.B., Rasmussen, I., Omø, K., Pedersen, S.M., Polny, J., Andersson, H., Andersson, T., Kämäräinen, V., Vilhu, O., Huovelin, J., Maisala, S., Morawski, M., Juchnikowski, G., Costa, E., Feroci, M., Rubini, A., Rapisarda, M., Morelli, E., Carassiti, V., Frontera, F., Pelliciari, C., Loffredo, G., Martinez Nuez, S., Reglero, V., Velasco, T., Larsson, S., Svensson, R., Zdziarski, A.A., Castro-Tirado, A., Attina, P., Goria, M., Giulianelli, G., Cordero, F., Rezazad, M., Schmidt, M., Carli, R., Gomez, C., Jensen, P.L., Sarri, G., Tiemon, A., Orr, A., Much, R., Kretschmar, P. \& Schnopper, H.W., JEM-X: The X-ray monitor aboard INTEGRAL, A\&A, 411, L231-L238, 2003.

Lutz, D., ISO observations of the Galactic Centre, ESASP, 427, 623, 1999. 
Markoff, S., Falcke, H., Yuan, F. \& Biermann, P.L., The Nature of the 10 kilosecond X-ray flare in Sgr A*, A\&A, 379, L13-L16, 2001.

Marrone, D.P., Baganoff, F.K., Morris, M.R., Moran, J.M., Ghez, A.M., Hornstein, S.D., Dowell, C.D., Muoz, D.J., Bautz, M.W., Ricker, G.R., Brandt, W.N., Garmire, G.P., Lu, J.R., Matthews, K., Zhao, J.-H., Rao, R. \& Bower, G.C., An X-Ray, Infrared, and Submillimeter Flare of Sagittarius A*, ApJ, 682, 373-383, 2008.

Melia, F., The Galactic Supermassive Black Hole, Princeton University Press (41 William Street, Princeton, New Jersey 08540), 2007.

Melia, F. \& Falcke, H., The Supermassive Black Hole at the Galactic Center, ARAA, 39, 309-352, 2001.

Meyer, L., Schödel, R., Eckart, A., Karas, V., Dovčiak, M. \& Duschl, W.J., Kband polarimetry of an Sgr A* flare with a clear sub-flare structure, A\&A, 458, L25-L28, 2006.

Meyer, L., Schödel, R., Eckart, A., Duschl, W.J., Karas, V. \& Dovčiak, M., On the orientation of the Sagittarius A* system, A\&A, 473, 707-710, 2007.

Meyer, L., Do, T., Ghez, A., Morris, M.R., Witzel, G., Eckart, A., Bélanger, G. \& Schödel, R., A 600 Minute Near-Infrared Light Curve of Sagittarius A*, ApJ, 688, L17-20, 2008.

Morris, M., Tanner, A.M., Ghez, A.M., Becklin, E.E., Cotera, A., Werner, M.W. \& Ressler, M.E., Mid-Infrared Limits on Emission from Sagittarius A*, BAAS, 33, 841, 2001.

Pantin, E., Lagage, O.-P., Claret, A., Doucet, C., Kaufer, A., Käufl, H.-U., Pel, J.-W., Peletier, R.F., Siebenmorgen, R., Smette, A. \& Sterzik, M., Karas, V. \& Dovčiak, M., VISIR, a Taste of Scientific Potential, The Messenger, 119, 25-29, 2005.

Porquet, D., Predehl, P., Aschenbach, B., Grosso, N., Goldwurm, A., Goldoni, P., Warwick, R.S. \& Decourchelle, A., XMM-Newton observation of the brightest X-ray flare detected so far from Sgr A*, A\&A, 407, L17-L20, 2003.

Porquet, D., Grosso, N., Predehl, P., Hasinger, G., Yusef-Zadeh, F., Aschenbach, B., Trap, G., Melia, F., Warwick, R.S., Goldwurm, A., Bélanger, G., Tanaka, Y., Genzel, R., Dodds-Eden, K., Sakano, M. \& Ferrando, P., X-ray hiccups from Sagittarius A* observed by XMM-Newton. The second brightest flare and three moderate flares caught in half a day, A\&A, 488, 549-557, 2008.

Reid, M.J., The distance to the center of the Galaxy, ARA\&A, 31, 345-372, 1993.

Reid, M.J., Menten, K.M., Trippe, S., Ott, T. \& Genzel, R., The Position of Sagittarius A*. III. Motion of the Stellar Cusp, ApJ, 659, 378-388, 2007.

Reid, M.J., Broderick, A.E., Loeb, A., Honma, M. \& Brunthaler, A., Limits on the Position Wander of Sgr A*, ApJ, 682, 1041-1046, 2008.

Revnivtsev, M.G., Churazov, E.M., Sazonov, S.Y., Sunyaev, R.A., Lutovinov, A.A., Gilfanov, M.R., Vikhlinin, A.A., Shtykovsky, P.E. \& Pavlinsky, M.N., Hard X-ray view of the past activity of Sgr A* in a natural Compton mirror, A\&A, 425, L49-L52, 2004. 
Revnivtsev, M, Sazonov, S, Gilfanov, M, Churazov, E. \& Sunyaev, R., Origin of the Galactic ridge X-ray emission, A\&A, 452, 169-178, 2006.

Rybicki, G.B. \& Lightman, A.P., Radiative processes in astrophysics, New York, Wiley-Interscience, 1979. 393 p., 1979.

Rousset, G., Lacombe, F., Puget, P., Hubin, N.N., Gendron, E., Fusco, T., Arsenault, R., Charton, J., Feautrier, P., Gigan, P., Kern, P.Y., Lagrange, A.-M., Madec, P.-Y., Mouillet, D., Rabaud, D., Rabou, P., Stadler, E. \& Zins, G., NAOS, the first AO system of the VLT: on-sky performance, SPIE, 4839, 140-149, 2003.

Schödel, R., Ott, T., Genzel, R., Hofmann, R., Lehnert, M., Eckart, A., Mouawad, N., Alexander, T., Reid, M.J., Lenzen, R., Hartung, M., Lacombe, F., Rouan, D., Gendron, E., Rousset, G., Lagrange, A.-M., Brandner, W., Ageorges, N., Lidman, C., Moorwood, A.F.M., Spyromilio, J., Hubin, N. \& Menten, K.M., A star in a 15.2-year orbit around the supermassive black hole at the centre of the Milky Way, Nature, 419, 694-696, 2002.

Schödel, R., Eckart, A., Mužić, K., Meyer, L., Viehmann, T. \& Bower, G.C., The possibility of detecting Sagittarius $A^{*}$ at $8.6 \mu \mathrm{m}$ from sensitive imaging of the Galactic center, A\&A, 462, L1-L4, 2007.

Stolovy, S.R., Hayward, T.L. \&Herter, T., The First Mid-Infrared Detection of a Source Coincident with Sagittarius A *, ApJ, 470, L45, 1996.

Strüder, L., Briel, U., Dennerl, K., Hartmann, R., Kendziorra, E., Meidinger, N., Pfeffermann, E., Reppin, C., Aschenbach, B., Bornemann, W., Bräuninger, H., Burkert, W., Elender, M., Freyberg, M., Haberl, F., Hartner, G., Heuschmann, F., Hippmann, H., Kastelic, E., Kemmer, S., Kettenring, G., Kink, W., Krause, N., Müller, S., Oppitz, A., Pietsch, W., Popp, M., Predehl, P., Read, A., Stephan, K.H., Stötter, D., Trümper, J., Holl, P., Kemmer, J., Soltau, H., Stötter, R., Weber, U., Weichert, U., von Zanthier, C., Carathanassis, D., Lutz, G., Richter, R.H., Solc, P., Böttcher, H., Kuster, M., Staubert, R., Abbey, A., Holland, A., Turner, M., Balasini, M., Bignami, G.F., La Palombara, N., Villa, G., Buttler, W., Gianini, F., Lainé, R., Lumb, D. \& Dhez, P., The European Photon Imaging Camera on XMM-Newton: The pn-CCD camera, A\&A, 365, L18-L26, 2001.

Tagger, M. \& Melia, F., A Possible Rossby Wave Instability Origin for the Flares in Sagittarius A*, ApJ, 636, L33-L36, 2006.

Telesco, C.M., Davidson, J.A. \& Werner, M.W., 10-30 Micron Maps of the Central 5 Parsecs of the Galaxy: Heating of the Cavity and Neutral Gas Disk, ApJ, 456, 541, 1996.

Trap, G., Falanga, M., Goldwurm, A., Bozzo, E., Terrier, R., Ferrando, P., Porquet, D., Grosso, N. \& Sakano, M., Bursting behavior of the Galactic Center faint X-ray transient GRS 1741.9-2853, A\&A, 504, 501-510, 2009.

Trippe, S., Paumard, T., Ott, T., Gillessen, S., Eisenhauer, F., Martins, F. \& Genzel, R., A polarized infrared flare from Sagittarius A* and the signatures of orbiting plasma hotspots, A\&A, 375, 764-772, 2007.

Tsuboi, M., Miyazaki, A. \& Tsutsumi, T., Flare of SGR A* at Short Millimeter Wavelengths, ASPCS, 186, 105, 1999. 
Turner, M.J.L., Abbey, A., Arnaud, M., Balasini, M., Barbera, M., Belsole, E., Bennie, P.J., Bernard, J.P., Bignami, G.F., Boer, M., Briel, U., Butler, I., Cara, C., Chabaud, C., Cole, R., Collura, A., Conte, M., Cros, A., Denby, M., Dhez, P., Di Coco, G., Dowson, J., Ferrando, P., Ghizzardi, S., Gianotti, F., Goodall, C.V., Gretton, L., Griffiths, R.G., Hainaut, O., Hochedez, J.F., Holland, A.D., Jourdain, E., Kendziorra, E., Lagostina, A., Laine, R., La Palombara, N., Lortholary, M., Lumb, D., Marty, P., Molendi, S., Pigot, C., Poindron, E., Pounds, K.A., Reeves, J.N., Reppin, C., Rothenflug, R., Salvetat, P., Sauvageot, J.L., Schmitt, D., Sembay, S., Short, A.D.T., Spragg, J., Stephen, J., Strüder, L., Tiengo, A., Trifoglio, M., Trümper, J., Vercellone, S., Vigroux, L., Villa, G., Ward, M.J., Whitehead, S. \& Zonca, E., The European Photon Imaging Camera on XMM-Newton: The MOS cameras : The MOS cameras, A\&A, 365, L27-L35, 2001.

Ubertini, P., Lebrun, F., Di Cocco, G., Bazzano, A., Bird, A.J., Broenstad, K., Goldwurm, A., La Rosa, G., Labanti, C., Laurent, P., Mirabel, I.F., Quadrini, E.M., Ramsey, B., Reglero, V., Sabau, L., Sacco, B., Staubert, R., Vigroux, L., Weisskopf, M.C. \& Zdziarski, A.A., IBIS: The Imager onboard INTEGRAL, A\&A, 411, L131-L139, 2003.

Wang, Q.D., Lu, F.J. \& Gotthelf, E.V., G359.95-0.04: an energetic pulsar candidate near Sgr A*, MNRAS, 367, 937, 2006.

Westergaard, N.J., Kretschmar, P., Oxborrow, C.A., Larsson, S., Huovelin, J., Maisala, S., Martínez Núnez, S., Lund, N., Hornstrup, A., Brandt, S., Budtz-Jørgensen, C. \& Rasmussen, I.L., JEM-X science analysis software, A\&A, 411, L1-L6, 2003.

Winkler, C., Courvoisier, T.J.-L., Di Cocco, G., Gehrels, N., Giménez, A., Grebenev, S., Hermsen, W., Mas-Hesse, J.M., Lebrun, F., Lund, N., Palumbo, G.G.C., Paul, J., Roques, J.-P., Schnopper, H., Schönfelder, V., Sunyaev, R., Teegarden, B., Ubertini, P., Vedrenne, G. \& Dean, A.J., The INTEGRAL mission, A\&A, 411, L257-L260, 2003.

Yuan, F., Quataert, E. \& Narayan, R., Nonthermal Electrons in Radiatively Inefficient Accretion Flow Models of Sagittarius A*, ApJ, 598, 301-312, 2003.

Yuan, F., Quataert, E. \& Narayan, R., On the Nature of the Variable Infrared Emission from Sagittarius A*, ApJ, 606, 894-899, 2004.

Yusef-Zadeh, F., Bushouse, H., Dowell, C.D., Wardle, M., Roberts, D., Heinke, C., Bower, G.C., Vila-Vilaró, B., Shapiro, S., Goldwurm, A. \& Bélanger, G., A Multiwavelength Study of Sgr A*: The Role of Near-IR Flares in Production of X-Ray, Soft $\gamma$-Ray, and Submillimeter Emission, ApJ, 644, 198-213, 2006a.

Yusef-Zadeh, F., Roberts, D., Wardle, M., Heinke, C.O. \& Bower, G.C., Flaring Activity of Sagittarius A* at 43 and $22 \mathrm{GHz}$ : Evidence for Expanding Hot Plasma, ApJ, 650, 189-194, 2006b.

Yusef-Zadeh, F., Wardle, M., Heinke, C., Dowell, C.D., Roberts, D., Baganoff, F.K. \& Cotton, W., Simultaneous Chandra, CSO, and VLA Observations of Sgr A*: The Nature of Flaring Activity, ApJ, 682, 361-372, 2008. 
Yusef-Zadeh, F., Bushouse, H., Wardle, M., Heinke, C., Roberts, D. A., Dowell, C. D., Brunthaler, A., Reid, M. J., Martin, C. L., Marrone, D. P., Porquet, D., Grosso, N., Dodds-Eden, K., Bower, G. C., Wiesemeyer, H., Miyazaki, A., Pal, S., Gillessen, S., Goldwurm, A., Trap, G. \& Maness, H., Simultaneous Multi-Wavelength Observations of Sgr A* on 2007 April 1-7, Accepted for publication in ApJ, arXiv:0907.3786.

Zhao, J.-H., Bower, G.C. \& Goss, W.M., Radio Variability of Sagittarius A*-a 106 Day Cycle, ApJ, 547, L29-L32, 2001.

Zhao, J.-H., Young, K.H., Herrnstein, R.M., Ho, P.T.P., Tsutsumi, T., Lo, K.Y., Goss, W.M. \& Bower, G.C., Variability of Sagittarius A*: Flares at 1 Millimeter, ApJ, 586, L29-L32, 2003.

Zylka, R., Mezger, P.G., Ward-Thompson, D., Duschl, W.J. \& Lesch, H., Anatomy of the Sagittarius A complex. 4: SGR A* and the Central Cavity revisited, A\&A, 297, 83-97, 1995. 